\title{
STATIC STRAINS IN HIGH TENSION CIRCUITS AND THE PROTECTION OF APPARATUS.
}

\author{
BY PERCY H. THOMAS.
}

It is the purpose of the present paper to discuss the so-called "static effects" in high tension circuits, especial attention being given to the disturbances produced by lightning, switching, grounding and the like. Some discussions of particular phases of the principles involved have been published from time to time, but so far as has come to the attention of the author, no comprehensive treatment of the subject of a non-mathematical character has yet appeared. On this account a general view of the question without mathematical complication will probably prove of interest to superintendents and station managers as well as to electrical engineers. Necessarily much of the matter in the paper will not be new, but it will be found that the old principles when applied to commercial circuits yield quite nove1 and important results.

It is scarcely necessary to state that static strains, though not perhaps particularly destructive of themselves, may indirectly cause the loss or temporary disabling of expensive and important apparatus; and more is involved than the loss of the apparatus. One of the principal aims, if not the chief aim of the superintendent of a modern electric transmission system, for the accomplishment of which he will make great sacrifices, is. to keep his service continuous. Static disturbances in such systems, when not properly controlled, are a constant menace to the continuity of the service. The prominence of static effects is one of the chief distinguishing characteristics of high tension operation. The importance of " static " is not emphasized solely on theoreti- 
cal grounds. Plants in actual operation have suffered both damage to apparatus and loss of prestige on account of insufficient static protection.

The paper will be divided into two parts.

I. Nature, Causes and Effects of Static Disturbances; and

II. The Protection of Apparatus and the "Static Interrupter.'

Part I.-Nature, Causes and Effects of Static DisturbANCES.

INTRODUCTORY.

The term "static" is certainly a misnomer, as the most prominent characteristic of the phenomena thus designated is the abrupt and violent nature of their discharges of electricity. However, this group of phenomena is so clearly defined and is so generally recognized by this name that in the absence of a better term of general acceptance its use will be continued. A new term, however, is very greatly to be desired, and as the phenomena described are essentially those of wave motion, the author recommends the word "undic" as a substitute for "static."

For the present discussion "static" (or "undic") will be taken to include those changes of potential and waves of current of an abrupt nature which result from the transfer of the electrostatic charges of a system from point to point. Such actions are not directly produced nor controlled by the generator. I say " not directly," for, of course, practically all electrical phenomena in a circuit, except those due to lightning, are produced at least indirectly by the generator.

Every electric circuit may be viewed in two ways. It may be regarded (as in dealing with the transmission of power) as a closed circuit containing resistance and inductance, and carrying a certain current produced by a certain e.m.f., or it may be regarded (as in dealing with static) as a large insulated "conductor" (as the term is used in treatises on static electricity) of peculiar form. That is, the circuit performs a double function. It transfers useful energy and at the same time acts as an electric condenser. The first aspect, being the one of greatest practical importance, is the one almost universally considered. However, even in this case, with long high tension lines, it has been found necessary to give some attention to the e.s. (electrostatic) capacity of the circuit taken as an insulated " conductor." The 
second aspect of the circuit is the one to be here considered, and though of less importance than the first, it still deserves careful consideration on account of the serious difficulties static may cause in commercial work. As a matter of fact, since the electric circuit when operating is at the same time both a closed circuit carrying current and a charged insulated " conductor," the actual resultant condition of the system is a combination of these two states.

There are some points of difference, however, between the commercial electric circuit and the insulated " conductors" commonly used in the laboratory demonstrations of static electricity. In the first place the commercial circuit is very much the larger and is extended over greater distances, so that a sensible time is required for an electric impulse to pass from one end to the other. Consequently, two points on the same conductor may be momentarily at very different potentials. In the second place the amount of energy stored in the e.s. capacity of the commercial circuit is very much larger than that in the "conductors" of laboratory experiments. It should be noted that these points of difference are both difference of degree, not of kind.

It will be well at the outset to summarize briefly the most characteristic of the laws of static. These are all well known and of very general applicability, viz.:

LAws of Static.

I. A static discharge (which is merely a very sudden rush of electricity) encountering a choke coil in its path, experiences momentarily great opposition, i. e., the electricity is temporarily held back by the inductance of the coil, so that if the rush of electricity be of large volume and sufficiently sudden, a very great e.m.f. (neglecting losses of energy) will be exerted on the choke coil. This high e.m.f. is but momentary, however, as the choke coil after the first instant allows the electricity to pass at an accelerating rate which soon relieves the pressure. The high momentary pressure at the front of the choke coil causes a tendency for the charge to seek other paths. Such a phenomenon would be called " side flash " and is one of the considerations which led to the use of choke coils with lightning arresters. Lightning arrester choke coils are so placed that any static disturbance on the line must pass the choke coil to reach the apparatus whioh is being protected, and the arresters are placed on the line side of the choke coils, where the tendency to side flash is strongest.

As there is a tendency to side flash toward other objects, there 
must evidently also be a tendency to short-circuit the choke coil itself. Such short-circuits are by no means unusual when coils not designed to withstand them are exposed to static discharges,

Il. All conducting bodies have e.s. capacity or in other words are to a greater or less degree condensers. Whenever a conductor is at a potential different from a neighboring conductor, a charge of electricity appears in its surface principally on the side toward the other body. The amount of this charge equals the product of the e.s. capacity and the potential between the bodies. At the same time an exactly equal charge appears on the second body. A conductor may have capacity to two or more adjacent conductors at the same time; in this case its resultant charge is the sum of the separate charges.

The fact that a certain quantity of electricity, great or smail, is required to charge any conductor to any definite potential is very important in its bearing on long distance lines. Suppose, for instance, that a piece of apparatus at one end of a transmission line is to be raised to a certain potential by a sudden application of voltage at the other end. A sensible time will elapse before the necessary charge for the apparatus can reach the end of the long line. Consequently, the potential of the apparatus will not rise at the same instant as the applied e.m.f., but will remain unchanged until charge actually reaches it. In other words, the potential of a condenser cannot rise until the charge necessary to produce this rise has entered it.

The Iarge current required to charge the line wires of an extensive high tension system is well known. All switches, transformers, instruments and other apparatus actually connected to the high tension circuit also require charge, though of course much less.

The line wires have e.s. capacity both to the earth and to one another. The several charges corresponding to those different capacities though superposed on the wire, act independently of one another and must be so studied. Similarly with apparatus connected to the lines, not only will there be e.s. capacity in the high tension parts to ground and low tension parts, but also between different parts of the high tension windings themselves.

III. When a current is flowing through a circuit containing inductance, energy is stored in its magnetic field. The amount of this energy equals one-half the product of the inductance and the square of the current. This energy was obtained from the circuit when the current was started, and when the e.m.f. pro- 
ducing the current is removed, must be discharged back into the circuit before the current can stop. If the circuit be suddenly opened, a very high potential will be developed to keep the current flowing until this energy is fully discharged. Such is the case of the well-known rise of potential which results from suddenly opening the field circuit of a large generator. The direction of this "extra" e.m.f. is such as to continue the current flow.

Energy in a magnetic field is just as truly stored energy as that in the moving bullet, the stretched spring, or the raised weight. It may be adapted to useful purposes. For example, the energy stored in the magnetism in the core of an induction coil by the primary current, is discharged into the secondary to produce the high tension spark.

An idea of the amount of this energy in actual cases may be obtained from the following: One ampere flowing through a coil with an inductance of 1 henry (equivalent to $377 \mathrm{ohms}$ at 60 cycles) stores $\frac{1}{2}$ volt-ampere-sec $=\frac{1}{2}$ watt-second, $=\frac{1}{2}$ joule $=$ 0.367 foot-pounds.

The very sudden rushes of static electricity are no exception to this law; they produce magnetism and store energy which must be discharged into the circuit again before the currents can cease. This principle will be found to lead to important results.

Also, when a condenser is charged, energy is stored. This energy will be restored to the circuit again when the condenser is discharged. The amount of this energy is one-half the product of its e.s. capacity and the square of the voltage.

A condenser of one microfarad capacity charged to 1,000 volts potential stores $\frac{1}{2}$ a watt-sec. $=\frac{1}{2}$ joule, $=1.367$ footpounds. ${ }^{1}$

1. The transmission lines of a system like the Bay Counties Power Co. of Cal., with lines 150 miles long, have a capacity of about 3 microfarads. Operating at 40,000 volts, this will have $1 / 2 \times\left(3 \times 10^{-6}\right)(40,000 \times \sqrt{2})^{2}=$ 4,800 watt-secs $=4,800$ joules, $=3,500$ foot-pounds of energy stored in its e.s. capacity when fully charged. The charging current is 45 amperes at 40,000 volts, 60 cycles. Therefore, the rate of supply of energy to the line from the generator and absorption from the line by the generator is $45 \times 40,000 \times 2 \times \frac{1}{\tau}=1,150,000$ watts, $=1,150,000$ joules per $\mathrm{sec}_{\text {. }}=$ 843,000 foot-pounds per sec. The generator supplies current continuously to the line for $\frac{1}{4}$ cycle and then receives it back again for the next $\frac{1}{4}$ cycle. Therefore, the energy delivered or received in a half alternation is the energy stored in the capacity of the line, $=\frac{45 \times 40,000 \times 2}{\tau \times 60 \times 4}=4,800$ wattsecs. $=4,800$ joules $=3,500$ foot-pounds, as before. 
The energy of an electric current may thus be stored in two ways: either in a magnetic field produced by a current, or by the storing of the electricity itself. In its former state the energy may be regarded as kinetic and in the latter as potential.

\section{Electric Circuit as an Insulated Conductor.}

It has been stated that an electric circuit may be considered as an insulated conductor of a peculiar form. It is rather an aggregation of conductors of various forms. In general a high tension circuit consists of the following parts:

(a) High tension windings of transformers, raising and lowering.

(b) Transmission wires.

(c) High tension switches, instruments, lightning arresters, station wiring and similar auxiliary apparatus.

(a) The high tension transformer windings are in the form of coils, and are therefore from the static point of view, choke coils. On account of the very large number of turns their choking power is very great (choking power in general, being proportional to the squnre of the numbers of turns). The high tension windings have e.s. capacity also, as they lie close to the low tension windings and to the core, that is, the high tension winding will have static charges on the surface opposite the low tension winding and opposite the core. There is as well, capacity between turns and between coils of the high tension windings. The e.s. capacity of these transformer coils is of a rather complicated nature, for it is distributed along at different points on the winding, and is not concentrated at one point as in an ordinary condenser. Therefore, the charge for the different portions of the windings must pass through different lengths of wire and different amounts of inductance and resistance. The same is true of both raising and lowering transformers.

(b) The transmission wires have e.s. capacity with one another and with the earth, so there are two or more charges superposed on each wire-each of which may act independently of the others. This capacity, though comparatively small for short lengths of wire is, as a whole, large, on account of the great length of the lines. These wires have inductance also, like the capacity; small for short lengths, but quite considerable when the line is taken as a whole

On the line wires, as in the high tension transformer windings, the e.s. capacity and inductance are distributed, so that when 
voltage is applied to the line, different points receive charging current at different instants of time, since the charge for various points must flow through different lengths of line. In fact, the transmission wires may be considered as a succession of choke coils and condensers in series, each very small, but very great in number, as shown in Fig. 1.

In addition to the e.s. capacity of the line wires, there is a certain amount of capacity on each insulator. The amount is very small, and may be considered a part of the line capacity.

(c) Switches, instruments, lightning arresters, station wiring, and similar auxiliary apparatus have a certain amount of e.s. capacity to surrounding objects, but not usually as much as the high tension windings of transformers. This part of the system has also comparatively little inductance and needs no further discussion here.

In addition to e.s. capacity and inductance, all parts of the high tension system have more or less ohmic resistance. The

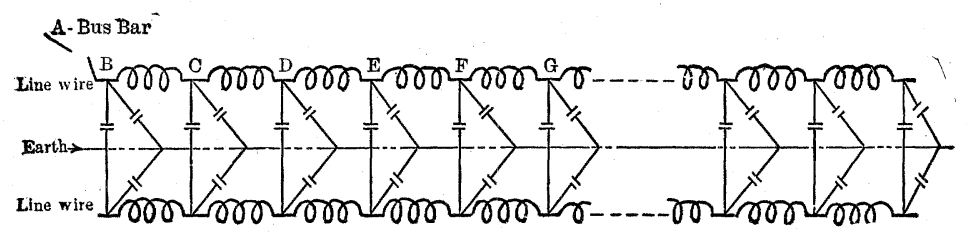

FIG. 1.-Diagram illustrating Inductance and Capacity of a Long Single Phase Transmission Line.

effect of this resistance is to retard the current (static or normal) in whatever direction it flows, and to change part of the electric energy into heat.

A certain amount of current leaks from the line at insulators and at all points where insulating material touches the circuit, since no insulation is perfect. Even the air conducts away some charge when the voltage is extremely high. Taken altogether, the result of these leakages is small under normal conditions, and may be neglected in this discussion.

Every point of a high tension circuit at any instant has a perfectly definite potential, which is in general different from the potential of other parts of the circuit and of adjacent objects. Consequently each point has at each instant a definite static charge, often different from that of other points. As (with alternating currents) the potential of each point is varying from instant to instant, these charges are continually changing and the changing charges cause a current in the circuit properly called 
charging current. Neither this charging current nor the static charges have any connection with the useful current except as the latter may influence the static potential of some point of the circuit. The total current in the circuit at any instant is the sum of the work current and charging current, if they are in the same direction, or their difference if they are in opposite directions.

When the potentials of all points of a circuit are determined directly by the electromotive force of the generator, i.e., during normal operation, all changes of potential are slow (compared with static changes of voltage) and allow sufficient time for the necessary changes in the static charges at various points to be accomplished without serious opposition from the inductance of the circuit. Such changes of potential and charges as these, however, are not the subject of this paper. Its principal object is the discussion of changes of potential of a more abrupt nature which are not directly produced by the e.m.f. of the generator; the latter may cause violent and sudden alternations of the static charges and dangerous local potential strains. However, before taking up the more complicated case of the abrupt changes, it will be best to consider a little more fully, the distribution of potential and charge in some of the simpler cases of normal running, viz.:

Static Charges on a Symmetrical Single-phase Transmission Line Open-Circuited at the End, and Charged by a Direct Current Generator.-In this case both lines have the same e.s. capacity to the earth. There is also capacity between the line wires. On the positive line is a positive charge composed of two parts, one due to its capacity to earth and the other to its capacity to the other wire; similarly on the negative wire is an equal negative charge composed of two parts. These charges remain constant as long as the e.m.f. of the generator is constant.

If, however, the generator be an alternator, the charges change from positive to negative with the voltage, remaining equal.on the two wires at all times. This interchange of charges requires a flow of current from line to line through the generator.

When the same line is loaded at the farther end no material change is produced in the line charges. Since different parts of the high tension winding of the motor or transformer or other apparatus constituting the load, are at potentials varying all the way from one-half line voltage positive to one-half line voltage negative, the charges on these points will vary in a similar manner. These charges are supplied from the generator through the line. The charges for the inner parts of the winding of the load 
apparatus must flow through the outer parts of the winding as well as through the line.

Same Line with One Leg Grounded.-Since direct current is practically never used in high tension work it will be omitted from further discussion here. The case of a grounded line is of importance not as a practical operating condition, but on account of the harmful results which may follow accidental grounds. The potential of one line wire becomes the same as that of the earth, while that of the other is full line voltage, double its value with circuit ungrounded. The potential between the wires being maintained by the generator, remains unchanged. Of the two component parts constituting the charge on the ungrounded wire, the part due to the e.s. capacity between the wires in undisturbed by the grounding, since the voltage between wires is unchanged, but the part due to the capacity to earth is doubled since the potential of the line above the earth is doubled. On the grounded line the part of the charge due to the e.s. capacity between wires is unchanged-the part of charge due to capacity to earth becomes zero, since the potential to earth is zero. The charge which appears on the earth opposite the ungrounded wire and which is equal in amount to the part of that line's charge that is due to capacity with the earth, being produced by the generator, must flow to earth at the point where the circuit is grounded. On a large system the e.s. capacity of the lines would be sufficient to make this current quite large. In such a case the current may burn off the line wire at the grounded point if the contact with the earth is imperfect. This fact has a very direct bearing on the question as to whether it is possible to operate a large high tension system with one wire accidentally grounded.

System with Nentral Point Grounded.-In a symmetrically arranged ungrounded system either single phase or polyphase, a neutral point may be defined as any point which is at the earth's potential. There may be more than one neutral point in a circuit as shown in Fig. 2, where $a b c d$ are neutral points.

Since the neutral point is at the earth's potential, grounding this point makes no difference in the potential or charge of any point of the circuit, and no current flows over the grounding wire. If the circuit becomes unbalanced, for example, by a high resistance ground on one wire-there is a tendency for the neutral point to take a potential different from that of the earth, and a current will then flow over the grounding wire, which will be sufficient to keep the neutral point at earth's potential. If there 
be a dead ground on one wire the earth connection at the neutral point completes a short-circuit. If the neutral point is not grounded when the system becomes unbalanced, one or more lines will take a potential higher above the earth than in the balanced condition, and the other line or lines a potential lower than before. Therefore, comparing the two methods of operation-with the neutral grounded, if a " dead " ground is made on any line wire, a short-circuit is produced through the ground connection at the neutral point; with the neutral undergrounded a ground on a line wire means approximately twice the previous potential between line and ground on one or more wires, though no short-circuit is produced. The advantage of a grounded neutral is the preventing of increased potential over the earth in case of

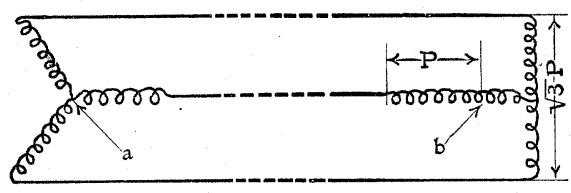

3 Phase Star

3 Phase-2 Phase

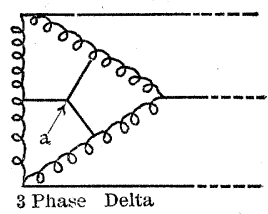

3 Phase Delia
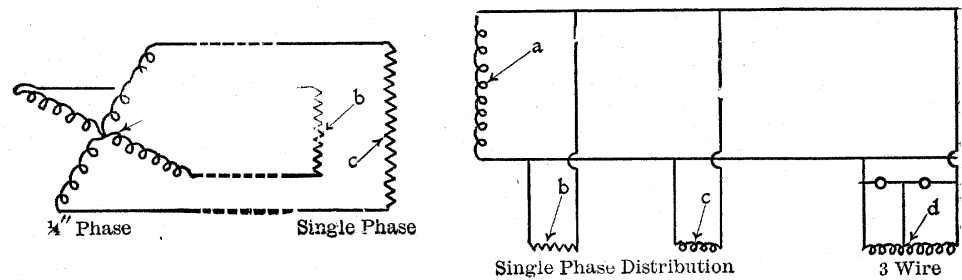

FIG. 2.-Neutral Points.

an accidental ground, its disadvantage is the fact that one such accidental ground, instead of two, causes a short-circuit.

We may now consider some of the abrupt changes of potential not directly produced by the generator.

1. Connecting "Dead" Transformer to Live Line.-Fig. 3 represents the portion of a high tension transformer coil connected to the line terminal. The circles indicate wires. The coil may have one or more turns per layer. The numbers on the circles indicate the order in which the current passes through the turns of the winding. The low tension winding lies adjacent to the high tension and the small condensers $a b c d$, etc., shown dotted, indicate the e.s. capacity of the corresponding wires to the adjacent low tension coils. 
Before either terminal of the transformer is connected to the line, all parts of the high tension winding are at earth's potential. As the first line switch is being closed at $s$, there is no change in the potential of the transformer winding until a spark passes between the switch jaws. Then instantly the potential of the transformer terminal is raised to the potential of this line wire. The rest of the high tension transformer winding also takes the potential of this line wire as soon as (but not sooner than) the necessary charge can reach $b-c-d-e$, etc. Now, the charges for $b-c-d-e$, etc., must flow through a considerable amount of inductance, which will require a length of time which is very short but still sensible. It is clear that during this short but definite period, after the terminal has reached its full potential and before there has been sufficient time for charge to reach the

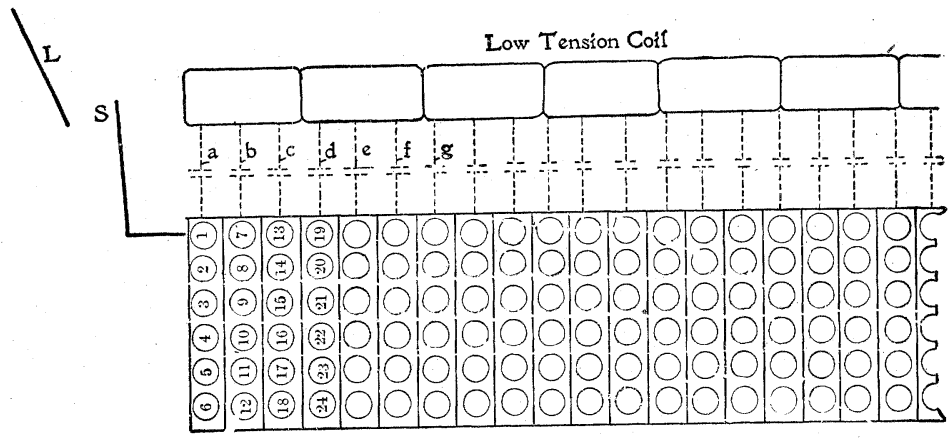

High Tension Coil

FIG. 3.-Diagram Illustrating e.s. Capacity of a High Tension Transformer Coil also Charging from a Live Line.

inner layers, for example, $f$ and $g$, etc., a difference of potential exists between the outer and inner layers of the winding, which is equal to the full e.m.f. of the line wire above the earth. If the insulation of the winding is too weak, or the line potential is sufficiently high, this momentary difference of potential will cause a spark to pass over the surface of the coil or through its insulation. This spark contains a very small amount of electricity, for as soon as the wires, $f, g$, etc., are charged up to line potential, the voltage between the inner and outer layers vanishes. The only result is the almost harmless passage of a nearly invisible spark.

Very soon after the closing of the first line switch the whole transformer winding will have assumed the potential of the first line wire, that is, just before the closing of the second line sw tch 
the second transformer terminal is at the potential of the first line. As the second switch is closed, a spark passes, and the potential of the second terminal of the transformer which has up to the instant been at the potential of the first line, is suddenly changed to that of the second line-a very abrupt change. Then, as before, during the period required for the necessary charge to penetrate to the inner turns, a very high potential difference is impressed on the outer portion of the coil. The momentary strain on the insulation of the coil is greater when the second switch is closed than the first, for the first transformer terminal experienced an abrupt change only from earth's potential to line potential, while the second was changed from the potential of one line to that of the other line, which is nearly double the potential from the ground.

There is another very important difference between the effects of closing the first and second switches in connecting a "dead" transformer to a live line. In the case of closing the first, as already stated, if the momentary strains break down the insulation, only sufficient current flows through this break to charge up the inner layers of the transformer coil. This is a very small quantity and can do comparatively little injury to the coil, especially if it be oil-insulated. In the second case the amount of current passing in the static spark is not very materially greater than in the first case. But when the insulation between turns is momentarily broken down by this small spark, there flows through the break a certain amount of current due to the e.m.f. impressed by the generator on each turn of the coil. A1though the static spark of itself would be but momentary, yet the current supported by this impressed or " normal " e.m.f. of the circuit may be able to hold the arc and continue indefinitely, destroying the whole coil if not interrupted. I say may be able to hold an arc, for such an arc may or may not be held, according to the circumstances of the case. If the static spark passes when the normal e.m.f. is nearly zero, or if the transformer be not able to supply much current in short-circuited turns, etc., then the chance that a permanent arc will be established is small. Sparks or flashes have actually been observed in the winding of high tension transformers at the time of lightning discharges, showing actual temporary holding of an arc.

The factors which determine the minimum number of layers upon which excessive momentary potential will be impressed are, chiefly, the inductance and e.s. capacity of the transformer coils 
and the abruptness of the change of potential of the terminal on the closing of the switch. The former determines the rate at which the charge can penetrate the coil, and the latter determines the time during which charging current may be passing into the coil before the full potential is reached on the terminal. The more abrupt the spark and the greater the capacity and inductance of the coils, the fewer the number of layers which will become charged before full terminal potential is reached, and the more severe will be the strain on insulation.

When switching is done on a high tension generator or a motor or any apparatus containing coils, a strain is brought in the windings near the terminals in exactly the same way as with transformer coils. There is also, usually, this same tendency for normal voltage to cause an arc to follow any momentary break in the insulation.

It is evident that the danger from this sort of switching is greater and greater for higher and higher voltages-it is of little importance on low voltages. Actually, injury of a serious nature to apparatus from this source is very much less than would be at first expected for several reasons:

(a) Insulating materials will stand much higher voltages of a static nature than of a continuous nature, such as those derived from generators.

(b) The passing of a static spark alone is usually by no means a serious matter and many circumstances may prevent a destructive arc from following the spark.

(c) It is only occasionally that the combination of circumstances arises which gives the severest conditions.

It has been assumed thus far that the potential of the line wires is unaffected by the switching operations. If, however, the connecting of the transformer to the line momentarily lowers the line potential at the switch, as will often occur, the abruptness of the static strain, and therefore its severity will be reduced.

This discussion which has been applied to switching on a transformer, is applicable when any coil is subjected to an abrupt change of potential in any part. For example, when a dead transmission line is connected to a live transformer the potential of the transformer terminals will usually be suddenly lowered very considerably for the moment, on account of the great excess of e.s. capacity in the line over the transformer coil. In this case the potential of the line which must be charged directly by the generator (and not from charge previously 
stored in the e.s. capacity of ocher lines) will rise very much more slowly, since current must pass through considerable inductance in generator and transformers. Sudden short-circuits, grounds, discharges of lightning arresters and similar disturbances all produce static strains more or less severe according to circumstances.

\section{FOURTH LAW OR STATIC.}

Before continuing the consideration of other cases of serious static it will be best to consider one or two more fundamental cases:

IV. When a condenser discharges suddenly through an inductive circuit containing comparatively little resistance, the current in the discharge path increases steadily until the condenser is completely discharged. At this time the current, in virtue of the magnetic field which it produces, stores nearly the full amount of the energy originally in the condenser, for there is little waste, as the resistance is assumed to be small.

But this current cannot become zero until all its energy is discharged into the circuit, which in this case means into the condenser; therefore the condenser is charged to its original voltage again, but this time in the opposite direction. At the instant all the energy is thus restored to the condenser (with reversed direction) the current is zero. But the condenser cannot remain charged with the discharge path closed, and will discharge again, repeating the same phenomena; that is, the charge oscillates backward and forward through the discharge circuit. If the resistance is not zero, a little energy is lost every time discharge occurs, so that each time the condenser is charged to a lower potential than the preceding time until the whole of the energy is finally transformed into heat. If now a condenser be charged through inductance, energy will be steadily stored in the magnetic field produced by the charging current (just as in the case of discharge) until the condenser has reached full potential. All this energy must be discharged into the condenser before the charging current can cease; that is, if there are no sensible resistance losses, the condenser will be charged momentarily to double potential. The condenser will then oscillate between double potential and zero until it finally settles down at the potential of the circuit from which it is charged, which occurs only as the oscillations are damped by the resistance of the circuit or by other losses. These phenomena may be made clearer by a mechanical analogy. 
The first case of the discharging condenser is similar to that of a pendulum which has been drawn aside and released; it oscillates about its final position of rest until its energy is all expended in friction. The second case may be compared to a weight supported by a spring. If the weight be dropped it will descend beyond the point at which it is finally to come to rest, stretching the spring to twice its final extension. The weight will then be drawn up again above the point of equilibrium, oscillating backward and forward until its energy is dissipated by friction. The inertia of the weight corresponds to the inductance of the charging circuit, and the elasticity of the spring corresponds to the capacity of the condenser.

Charging a Short Transmission Line.-Consider the charging of a short transmission line. The line may be considered to be a condenser; assume that it is being charged from bus bars rigidly maintained at constant potential. There will always be a certain amount of inductance in the path through which charging current must pass to reach the line, so that we have the case of the charging of a condenser through inductance with more or less resistance in the circuit. Therefore, when the line is connected, it rises to double potential (neglecting losses) and immediately starts to oscillate between this point and zero until the oscillation gradually dies out and leaves the line at normal potential. ${ }^{1}$ This means that at all points the insulation of the line will receive a strain of double potential. If, on the other hand, the bus bar from which the line is charged has not the capacity to deliver almost instantly the amount of electricity necessary to charge the line, the first effect of closing the switch will be not to raise the potential of the line to its full amount, but to make both line and bus bar take an intermediate potential so that the line comes up to normal potential by steps. Under these circumstances the maximum of the oscillations is materially reduced, and the line is not subjected to double potential. In the extreme case in which the bus bar has a comparatively small capacity to deliver current, the first effect of connecting the line is to bring the potential down to zero. In this case, if the line be charged from transformers, the outer portions of the windings are subjected to severe strains, as already explained. For a bus bar to be able to supply charging current to a "dead "line so quickly as not to have its potential momentarily dropped, it must have the

1. This rise of the line to double potential on charging was mentioned in Mr. Setinmetz's paper before the Institute August, 1901. 
necessary amount of electricity already stored in condensers connected directly to the circuit. In commercial plants the place of such condensers is supplied by other live lines connected to the bus bars.

The strain of double potential produced by charging a line has an interesting analogy. If a piece of metal be tested for tensile strength by suddenly applying a weight on the end, a duuble strain will be momentarily given the metal due to the slight motion of the weight allowed by the stretching of the test sample. In this case the inertia of the weight corresponds to the inductance through which the line is charged.

The discussion so far assumes that there are no losses of energy in resistance or in currents set up in adjacent bodies. Such losses, which always exist to some extent, tend to reduce both the amplitude and the number of oscillations.

\section{Charging a Long Transmission Line.}

In the case of a long transmission line, however, the line cannot properly be considered to be a simple condenser, for this is equivalent to the assumption that the line is so short that its inductance is practically zero, and in actual long lines such is not the case. To present such a line, however, we may take a succession of choke coils and condensers connected in series, as in Fig. 1. Consider a single line wire open circuited at the receiving end.

The " dead "line is to be charged at the end, $B$, from the high tension bus-bar, $A$. Assume that, as the switch at $A$ is closed, the point $B$ is instantaneously raised to full potential. If now the line extended no further than the condenser $C$, we should have the case of a short line which has just been discussed; that. is, the line will rise to double potential and oscillate until it finally settles down at normal potential. But since the line shown in Fig. 1 does not end at $C$, as soon as the potential of condenser $C$ begins to rise, current begins to flow to condenser $D$, and as $C$ rises higher and higher, more and more current will flow to $D$. The potential of $D$ then begins to rise, which starts current to $E$, etc. As $D$ rises slowly at first, $C$ reaches bus-bar potential before $D$, and similarly $D$ before $E$, etc. As soon as $C$ reaches this potential it remains constant. At the same instant that $C$ reaches bus-bar potential the current value in the coil between $B$ and $C$ reaches a maximum and becomes constant. Similarly with the currents in the other coils. This final current,which appears in more and more coils as the line charges up, supplies the charge that is being added constantly at those points where 
the potential is changing, to continue the process of charging, though the potential of $C$ remains constant, that of $D$ continues to rise until it reaches the same value as $C$, when it too becomes constant, though the potential of $E$ continues to rise, and so on along the line. Similarly with $E$ and $F$, but each successive condenser reaches its maximum a little behind those nearer the point $A$, so that the net result is a wave of e.m.f. starting at the point $B$ and passing along the line.

The general distribution of the potential of the line showing the wave form at short intervals of time after connecting on the line is shown in Fig. 4.

This wave passes along leaving the lines fully charged. If the line be infinitely long it will experience no further disturbance. and if there are no resistance or other losses the wave will pass along to an indefinite distance, keeping its form and raising in turn all parts of the line to the full bus-bar potential. If there be considerable losses of energy as the wave proceeds (as there usually are in any actual circuit) this wave will lose its shape somewhat, and will get feebler and feebler, until, if the line be long enough, it ceases to be perceptible. This dying away of intensity may be slow enough in commercial lines, so that a large part of the original intensity of the wave will remain when the end of the line is reached. Now, the end of the line is open circuited, and the wave of electricity can go no further and is reflected back. At the reflecting point the maximum potential reached is twice that of the wave.

On being reflected, the wave immediately starts back along the line, leaving it charged to double potential (assuming no losses), and finally reaches the starting point, $A$, where its energy is absorbed by the bus-bar. But the line cannot stay at double potential, and a second wave is sent along the line, bringing it back to normal potential again. This second wave will be reflected at the open end, and will return again, but this time dropping the potential of the line to zero until, as the wave reaches $A$ again, the line is practically in its initial condition. This wave, in turn, is absorbed by the bus-bars, and the whole series of operations gone over again and again. If, however, there are resistance and other losses, the wave is growing thinner and thinner all the time, and finally dies away, having only partly charged the line. As the wave dies down by the resistance losses, an infinite number of small supplementary waves are sent out, which complete the charging of the line. If there are no losses, however, no supplementary waves will be formed. 
$\underset{\text { Bus Bar }}{\text { A, }}$
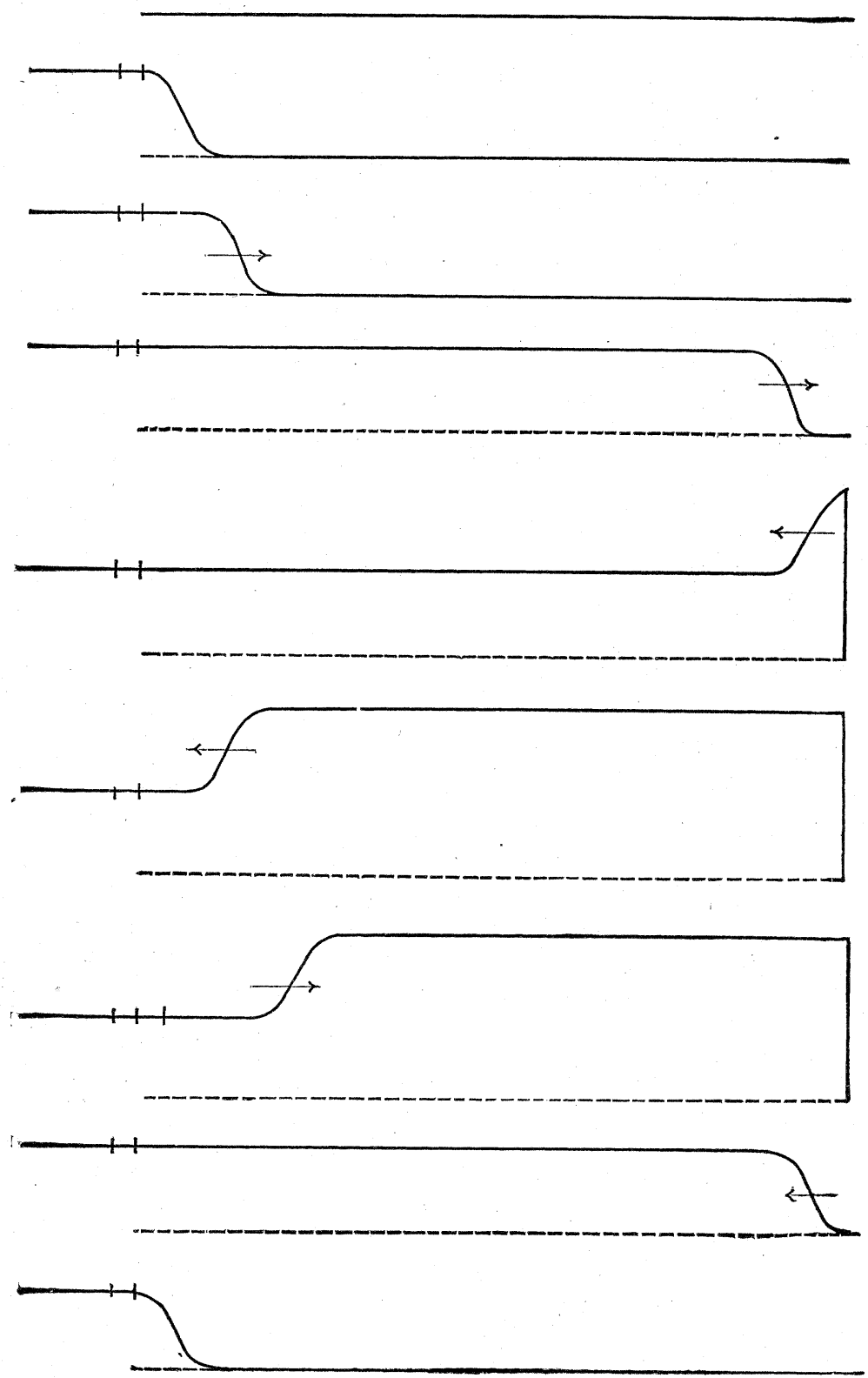

FIG. 4.-Charging Wave in a Long Transmission Line. 
It will thus be seen that the charging of a long line is much the same as a short line; in both cases the line oscillates between zero and double voltage until the losses cause it to settle down at normal potential. These wave changes are indicated in Fig. 4.

Actual tests on commercial transmission lines suddenly charged have shown a rise of potential much greater than normal at the end farthest from the switching.

Suppose a stretched flexible cord fastened at one end and held in the hand at the other. A quick motion of the hand sideways will send a loop or wave along the cord, which will pass to the end, will be reflected back and will finally reach the hand again. This wave is analagous to a static wave in the transmission line.

Charging a Branch Line.

Consider a line consisting of two parts-the more remote having a much smaller capacity and larger inductance than the nearer. When a wave starting from the beginning reaches the junction it will be partially reflected, since the whole charge of the large line cannot be crowded into the capacity of the small line, especially in view of the increased inductance of the new portion; that is, there will be a rise of potential at this point and a wave smaller than the outgoing wave will start back. As the original wave will not be wholly reflected, the potential at the juncture of the two circuits will not be double the charging voltage.

But a wave will also be sent forward into the second part of the line, which will have a crest as high as the maximum of the potential at the reflecting point. Therefore, at the end of the second line, where another reflection occurs, the crest of the last wave will be doubled. The resultant potential at this point will thus be very high, but not over four times the original voltage of the bus bar. It may be much less. This means that a branch line at the end of a main system (especially if the latter consist of two circuits in multiple) will receive a very severe shock at the farther end when a wave enters it from the main line. This is a case that may readily occur in actual plants and should be carefully considered. If there be a third line on the end of the second, leaving a still less capacity and still greater inductance, a wave will be formed in this line whose crest has the value of the maximum rise of the reflecting point at the end of the second section of line, and which will double its potential at the farther end as before. This last rise of potential has as its maximum theoretical limit eight times the original charging voltage, but 
would actually always be much less. This case is very unlikely to occur in actual circuits. The total energy in the waves in the second and third sections of lines is much less than in the original wave, but is at a higher potential.

To return to the consideration of the uniform circuit. It has been shown that when one end of a long line is suddenly raised. to a certain potential and maintained there, a wave of charge passes along the line, leaving it charged, and that when this wave reaches the open end of the line it is reflected and produces all along the line a potential double that of the bus bar. If, however, a sine e.m.f. be momentarily applied to the line so that the end of the line is not maintained steadily at the full potential as before, but is immeriately lowered, a wave will be found in the line as before, with the same maximum voltage but a different form. (See Fig. 5.) This wave leaves the line uncharged. The rise of potential at the reflecting point, however, is the same as long as the crest of the wave is the same, but in this case only the reflecting point receives double potential (assuming that only a single wave is sent into the line).

If a wave be started at one end in a very long, narrow trough of water, it will proceed the length of the trough and on reaching the end will be reflected and will rise up to double height at the reflecting point. This wave in the water is again analogous to the wave of charge passing along the line, leaving it unchanged and causing a double strain at the reflecting point.

In all cases of static effects the change in e.m.f. of the bus bars due to the generator is so slow that the generator e.m.f. may usually be considered constant while the static phenomena are taking place.

The length of the wave in the line is determined by two factors - the speed at which the charge passes along and the time required to bring the potential of the first point of the line to its full value; it is the extreme distance to which charge has penetrated when the charging potential becomes stationary.

If the line is shorter than the length of the wave no complete wave will be formed, but reflection will produce the same rise of double potential at the end. The speed at which the wave will pass along the line is inversely proportional to the square root of the product of the capacity and the inductance per unit length (unit length $=1$ earth quadrant $=6,200$ miles) - that is, to the time constant. But on air lines the waves always travel at approximately the speed of light, so that there is an inherent 
relation between the inductance and capacity of an air line which at first thought is very surprising. This relation is expressed in general by the equation $v=1 / \sqrt{L C}$, or $L=1 / v^{2} C$, where $v$ is the velocity of the wave in air.

Evidently the more sudden the disturbance the more likely is the formation of a complete wave. In the extreme case when the length of wave is very much longer than the line (as in the case when the voltage is applied to the line very slowly) there is practically no wave formed and we have the plain charging of a condenser. This is also the case considered in discussing the charging of a short transmission line.

If the source of e.m.f. from which the line is charged cannot maintain rigidly its potential during charging, the line will be charged up by steps as already explained. The result is that a weaker wave is obtained, followed by a second wave as the bus bar recovers its potential-which completes the charging of the line.

\section{Charging an Underground Cable.}

What has just been said of charging a line applies equally well to an underground cable, only the cable has a much larger e.s. capacity and a smaller inductance than the transmission line. If a wave be formed in charging the cable, this wave will be reflected at the end, causing double potential all along the cable as it returns to the starting point. If, however, the cable be so short that no wave be formed, the cable will be charged like a short line, that is, as though it were a condenser. As before, if a momentary impulse up and down be given the cable-instead of a steady charging voltage, a wave of the form shown in Fig. 5 wiii be produced, which causes double potential at the end of the cable, and there only when but one impulse is sent into the cable.

If a steady alternating e.m.f. is applied to a transmission line, waves will be sent along having crests of positive and negative values alternately and will be reflected at the end of the line one after another and return toward the starting point. When a returning positive crest meets an advancing positive crest, double potential will result at the point of meeting; similarly with two negative crests. When a positive and a negative crest meet, zero potential will result at the point of meeting. The result is the formation of nodes and loops in the line or cable, that is, points of zero and of double potential as shown in Fig. 6, where advancing waves are, shown full, reflected waves dotted, resultant nodes and loops in dot and dash. 
The waves sent into the line by the alternating e.m.f. are in continuous motion back and forward, but the nodes and loops are fixed in position though the loops vary in strength, alternating between positive and negative. This phenomenon is similar to the formation of nodes and loops in an organ pipe. There is always a loop or point of high potential at the reflecting point. The distance between two positive loops at any instant is the wave length of the moving waves.

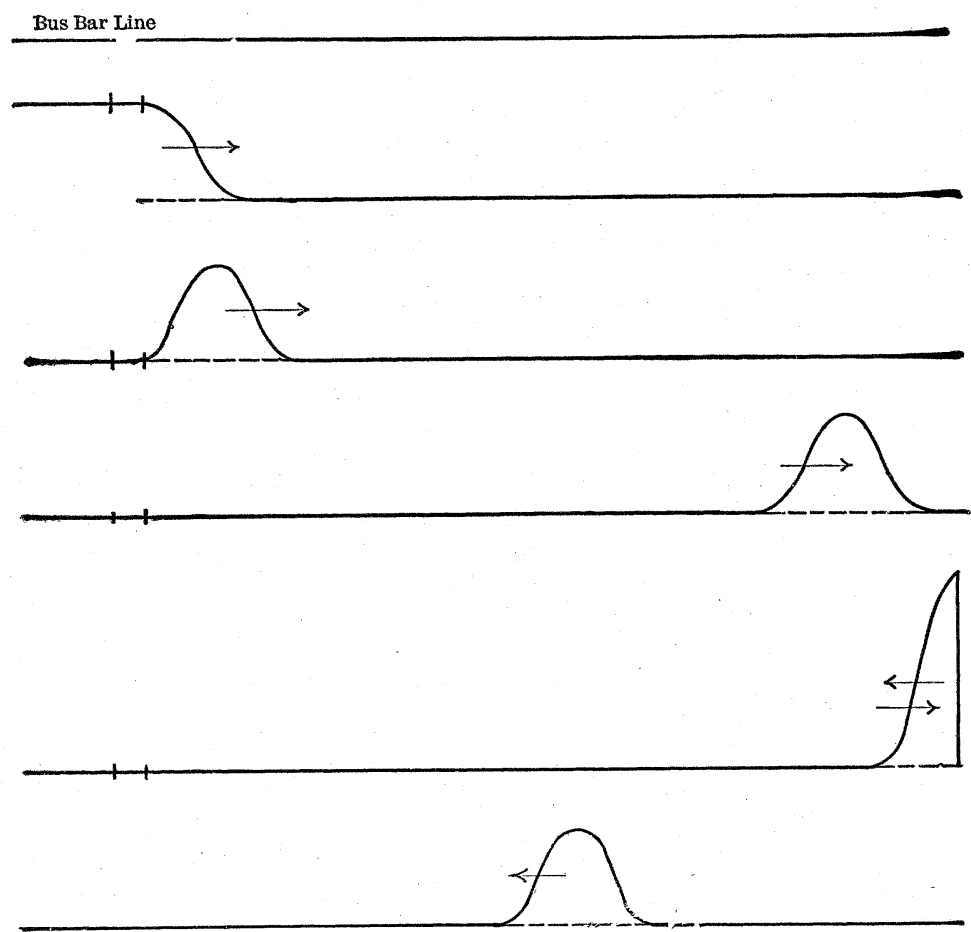

FIG. 5.-Charging Wave in Long Transmission Line.

To this point the lines and cables have been considered as open circuited at the farther end. Comparatively little difference will result in static effects, however, if they be closed through transformers at various points for the inductance of the transformer winding is so great that the comparatively large quantities of the charge on the line would not be materially lessened by the e.s. capacity of the transformers. In fact the transformer is subjected to a very severe strain without its being able to relieve the line. This strain is similar to that produced by connecting a " dead " transformer to a live line, since the rise of potential of 
the transformer terminal, due to reflecting the wave, may be very abrupt.

\section{Opening a High Tension Circuit.}

When opening a load current in a high tension line or cable no rise of potential will result unless the current be suddenly interrupted. With such an interruption, of course (as all commercial circuits contain considerable inductance), a rise of potential will result, its severity depending upon how suddenly the current is interrupted, how much magnetic energy is stored in the circuit, and how much e.s. capacity exists in the neighborhood of the break. This matter was treated by Mr. Steinmetz in his paper before the Institute last August. He there states that an axcessive rise of potential may result from the interruption of

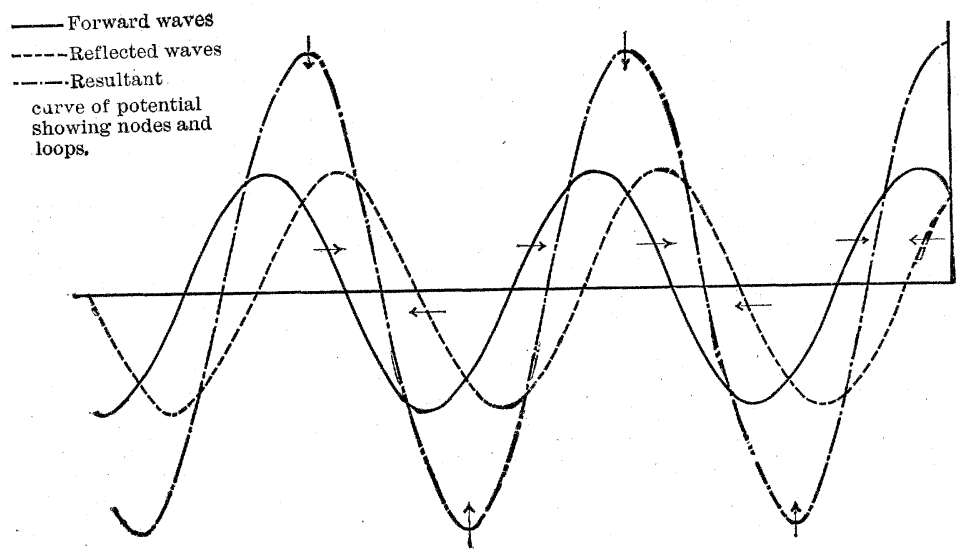

FIG, 6:-Nodes and Lopos in a Long Line or Cable.

short circuits. As a matter of actual experience, it is the opinion of the author that little rise of potential actually occurs from the opening of load or short circuit currents in commercial systems, as the resulting arc cannot drop out suddenly on account of the great amount of heat generated. There are often, however, other causes of static strains which may result from short circuits that occur at practically the same instant of time, and in some cases the results of one cause may be assigned to the other.

However, by opening an unloaded line or cable, it is entirely possible to obtain a rise of potential as follows: On opening a switch to cut out an unloaded line, the arc of the charging current tends to drop out when the current strength is zero, that is (since the unloaded line takes a leading current) when the voltage is a maximum; for as the line will momentarily hold its charge there 
is at this time little difference of potential between the line and bus bars, even after the switch is opened. The line is thus left charged when the switch is opened, while the potential of the bus bars is changing with the generator e.m.f. When the voltage of the generator has passed through one alternation there will ke a potential between the line and bus bars, which may be sufficient to cause the arc to establish itself again and recharge the line. The arc will again drop out when the current becomes zero, and this action may be repeated several times before the line is finally clear. The sharp crackling sound often accompanying the switching out of a high tension line or cable suggests this phenomenon. This recharging of the line will cause the formation of a wave and the consequent rise of potential as in the case of charging a line. Thus, pulling off an unloaded line may have the same effect as charging it so far as the static rise of potential is concerned.

In the case where one terminal of the single-phase generator is grounded and charging current to a line is opened at the other, a static wave may be produced in the line of double the intensity caused by charging the line "dead," that is, a wave of double line voltage, giving a maximum rise of potential of four times.

\section{Other Causes of Static Strains.}

In the previous discussion the author has endeavored to show that dangerous static effects result directly from very abrupt changes of potential. They may appear in parts of the circuit very remote from the exciting cause. These static strains are phenomena, such as would be expected to result from well known laws governing static electricity. In addition to the causes of abrupt changes of potential already discussed, a few more will be briefly described.

(a) Short Circuits.-A short circuit between the two line wires, when of a sudden nature, causes an abrupt reduction to zero of the potential of one or both lines at the foint of short circuit. This is the reverse of the case of charging a line. A wave is sent along the line, carsing a dropping of the potential, which of course has no tendency to ground apparatus. Nevertheless, this wave causes just as severe a strain on the insulation between turns of the transformer coils nextto line as a charging wave, for the potential of the transformer terminals is abruptly reduced. Since the inner layers cannot get rid of their charge immediately, there is a momentary potential across the coils between a high 
potential point in the inner layers and a low potential point at the. terminal.

(b) Grounds - Grounds are also a cause of abrupt change of potential and short circuits. The same strain on coils is produced as before, strongest on the side of the circuit where the ground occurs; also the ungrounded lines are raised to full potential above the earth. It is true that the rise of potential of the ungrounded lines is not necessarily as abrupt, since it is produced partly by the generator.

It must be remembered that the static waves produced in lines by grounds and short circuits may have their intensity doubled at a reflecting point, so that the short circuit strain on coils is doubly severe at such points. However, the strains to ground are not great in these cases, except on the ungrounded wires.

It must be remembered that when a wave is caused by grounding or charging a line that the abrupt change of potential may sometimes be only the voltage existing between line and ground in that system and not the full line voltage.

In much of the previous discussion, transmission lines have been assumed to be single-phase. The conclusions arrived at are nearly all applicable to polyphase lines as well, with occasional changes which will be evident on inspection.

(c). Lightning.-Lightning is the best known cause of static disturbances. Unlike grounds, short circuits and switching, lightning usually does not act directly on the circuit, but indirectly by induction. Its effects, however, are of the same nature as those already discussed.

In general, lightning may affect a transmission line in two ways; by induction and direct by stroke.

(1) A lightning discharge in the neighborhood of an electric circuit but not actually reaching the circuit acts upon it indirectly by a combination of static and dynamic induction. The actual intensity of the effect in the circuit depends on the energy of the lightning discharge, on the distance to the circuit, its form and its position relative to the discharge path of the lightning and upon other conditions.

Lightning discharges are usually of an oscillating nature, that is, the charge on the cloud oscillates backward and forward between cloud and earth several times, pausing at each end of its path in each oscillation. This oscillating charge acts inductively on any electric circuit which is in the neighborhood, first (static induction), when pausing at either end of its path (by attracting a 
charge of the opposite sign to the nearest point of the circuit), and second (electromagnetic induction), since the actual discharge is a current and sets up an opposing e.m.f. in any parallel conductor. As the lightning charge appears first at one end and then the other of its discharge path, it draws the charge on the line back and forth, and the e.m.f. in the line induced by the current in the discharge is in such a direction as to accelerate the motion of the attracted charge. Therefore, the effect of the lightning discharge is to cause a static wave or a series of static waves in the line. These waves are similar in general character to those produced by switching, etc., except that there is no limit to their maximum possible strength. They may produce either grounds on windings or short circuits in coils, as circumstances may determine. The static strains produced in a circuit by lightning have no direct relation to the generator voltage, but are determined by the form and location of the line, the protective devices and the nature of the lightning discharge.

(2) A lightning discharge may and occasionally does strike a line directly. In this case it is commonly supposed that nothing can save the apparatus from injury. This may or may not be the fact, according to circumstances. If the discharge strikes the line at some distance from the apparatus, there is a very good chance that no harm may be done, for the choking effect of the line is enormous for the extremely abrupt discharge of a direct stroke. That is, the discharge cannot pass down a long line quickly, and instead will pile up voltage at the point where the line is struck and finally make some direct path to earth, usually by jumping to a pole. This relieves the line and no severe shock is sent to the station. The escaping discharge may or may not destroy the pole, according to the nature of the pole and the intensity of the discharge. But although the chief discharge passes to earth directly there will be a wave sent along the line, which may or may not do damage according to circumstances; thus, in this case the arresters are not required to discharge the direct stroke.

On the other hand, if the stroke is near the apparatus it is probable that protection will not be obtained from any lightning arrester. Even though the arrester were, for the moment, a direct connection between line and ground, there would be sufficient resistance and inductance in the ground connection and the discharge path in the earth itself, to prevent an absolutely free discharge and the result would be that the lightning would 
find other paths to ground. The case of a direct stroke by lightning near a station very rarely occurs.

(d) Paralleling out of Phase.-One more case of static may be mentioned. When two lines are thrown in parallel out of step there is an abrupt change of potential produced when the second leg of the paralleling switch is closed. This voltage change may run up to double line voltage if the generators are directly opposite in phase. The same effects may be produced when two generators are running in parallel by opening all the poles of the paralleling-switch except one, for the machines soon get out of step, and a discharge may pass between circuits-either over arresters or elsewhere. In this case a synchronous motor or rotary converter will act as a generator for a time long enough to cause any possible damage to be accomplished.

The most frequent causes of dangerous static disturbances in high tension circuits have been discussed. It is evident, however, that anything which causes an abrupt change of static potential of considerable severity may cause a dangerous strain on apparatus.

Resonance.-Resonance does not, strictly speaking, come under the head of "static," but to show its relation to the actions already discussed, some attention will be given it. As already frequently stated, any condenser discharging through inductance (the resistance being small) has an oscillating discharge as a pendulum has an oscillating motion. If now an alternating e.m.f. be applied in the discharge circuit of the condenser in such a way that the e.m.f. alternates just as often as the condenser discharges and is always in the same direction as this discharge, the intensity of the oscillations will get greater and greater, just as the vibrations of the pendulum will get greater and greater if an alternating force is applied to it in such a way as always to increase its swing. There is no limit theoretically to the voltage that may be reached after a sufficient number of oscillations, if there are no losses. However, in any actual trial, the loss of energy in resistance and elsewhere gets greater as the oscillations get greater and will finally equal the energy put in by the alternating e.m.f. and thus stop the increase of voltage. Also, the exciting e.m.f. may slowly get out of step and so after building up the oscillations for a while, reduce them again. The increase of voltage may be stopped by a break down of insulation which may occur on acsount of the high voltage. That is, if we have an oscillating circuit (as, for example, a short transmission line) and an exciting 
cause of exactly the right alternations a very high voltage may result. The exciting cause may be either the generator e.m.f. or it may be some static e.m.f.; for example, another discharging condenser. In very few cases in actual installations, however, has it been directly shown that resonance has caused any serious rise of potential. There is no justification for hastily assigning resonance as the cause for unexplained static phenomena.

The number of complete oscillations per second in a circuit consisting of a choke coil and condenser $=1 / 2 \pi \sqrt{L C}$. So for the circuit to have a frequency of 60 cycles would require that the product of its capacity and inductance shall equal $1,(2 \pi 60)^{2}$. If the capacity equals 10 microfarads the inductance must equal $7 / 10$ henries, which is a large value. This shows that resonance with the generator e.m.f. is very unlikely in high tension lines. A condenser of $1 / 10$ microfarad with a coil of $1 / 1000$ of a henry has 16,000 complete oscillations per second.

When an alternating e.m.f. is applied to a long transmission line, however, the action is somewhat different from that of a simple condenser. The first alternation of the e.m.f. sends a wave down the line which is reflected back if the end is open. If the applied e.m.f. is just slow enough so that as it starts on its negative swing, the reflected wave is just beginning to arrive again at the starting point, its e.m.f. is added to that of the impressed voltage, and a second wave of double strength is sent along the line-when the next alternation is reflected back, the applied e.m.f. has reversed and again adds to the amplitude of the wave-thus just as with a simple condenser an excessive voltage will be built up if there are no losses. But suppose that the generator has three times this frequency. The applied e.m.f. will then have the same relative value, when the first wave reaches the beginning of the line after reflection, as when it has only one-third the frequency, though two other wave crestsone positive, one negative-have been sent into the line. The amplitude of the first wave will then be doubled as before and the same with the two succeeding waves. But by this time the first augmented wave has again been reflected and reached the starting point so that it has a further increase of amplitude. The same thing occurs if the applied e.m.f. has its frequency increased to five times its original value, only five waves are operated on in a group instead of three as in the previous case; similarly with 7 , 9 , etc., times the original frequency (which was such that in the time of one alternation a wave would pass the length of the line 
and return). Resonance of this type with a long line is much more likely to be met than with a simple condenser, for there are a great number of frequencies at which resonance may occur.

If the periodicity of the applied e.m.f. is not exactly right for resonance (this applies to long and short lines), there will still be a certain amount of rise of potential which will be less as the e.m.f. departs further from the proper frequency.

A summary of the conclusions so far reached follows:

(1) When a " dead" transformer is connected to a live line a strain may be produced on the layers of the coil next to the terminal, which may be as great as the line voltage.

(2) When a short line is charged suddenly from live bus bars, a momentary voltage rise may be produced which will be not more than double voltage.

(3) When a long line is charged a strain similar to that in the short line is produced, doubling the potential first at the end of the line and afterward along the whole length. This result assumes that the charging terminal of the line is abruptly raised to full normal potential and rigidly maintained there until the wave is fully formed; also that the losses are zero.

(4) The fundamental principles governing the charging of a cable are the same as those of a transmission line.

(5) Opening a light high tension circuit may be nearly the equivalent of charging the circuit, since before the line is completely freed from the bus bars it is often momentarily recharged several times.

(6) When a branch line of small e.s, capacity is supplied from a main line, the rise of potential at the farther end may be twice as great as at the end of the main line.

(7) In addition to switching, other causes of static strains are grounding, short circuit, lightning, etc., when these cause abrupt changes of potential.

(8) A rise of potential due to resonance is always possible, but generally improbable. It requires that there be an oscillating circuit and an exciting cause which must be of very nearly the same frequency as the oscillating circuit. When an alternating e.m.f. of high frequency is applied to a long line the danger that resonance will occur is usually greatest, as it may be produced by a large number of frequencies. 


\section{Part II.-Protection of Apparatus and the Static INTERRUPTER.}

Tracing of Damage Caused by Static.

Frorr the discussion in the first part of this paper, the conclusion seems warranted that some sort of static protection for apparatus in high tension circuits is very desirable. This fact has, indeed, long been almost universally recognized in a partial way, as is shown by the wide use of the lightning arrester. But the lightning arrester as commonly found falls far short of affording complete protection, although it has, in fact been quite effective in preventing grounds in apparatus (its only avowed object). As every person familiar with the operation of high tension electrical apparatus knows, short circuits as distinguished from grounds are a much more frequent cause of difficulty than grounds proper. Now, lightning arresters have little effectiveness for preventing short circuits, since the short circuit, when caused by static, is usually the result of the concentration on a small porton of the coil of a voltage, not necessarily great with respect to the line voltage, but perhaps very great with respect to the normal voltage of the portion of the coil where the failure occurs. Some protection, however, has frequently been secured by the use of choke coils in addition to the lightning arresters; but these have often been used in an aimless way, and have sometimes been omitted in very high tension circuits, where they are the most necessary.

The chief reason why static protection has not received more careful consideration (although the lightning arrester proper has been long in commercial use) is the very great difficulty of tracing the effects of static to their true cause. In the first place, very little is generally known as to what causes static strains and as to whereabouts in the circuit they are to be expected, or as to what sort of injuries they inflict. The direct damage from an excessive static strain is usually limited to the injury that can be caused by a spark carrying nothing but charging current to some small portion of the apparatus. Such an injury would be invisible to the eye. A great many repetitions would be required before an evident defect would appear.

In the second place when trouble develops from a static cause it is usually made manifest by an arc supported directly by the generator. This arc, of course, causes a great amount of burning of insulation and fusing of copper, which destroy all evidence of 
the original cause of the failure. Also at such a time usually little note is taken of attendant circumstances which could throw some light on the cause of the trouble. Most engineers will appreciate the great difficulty of getting afterwards the exact sequence of events in any breakdown. Again, sometimes a severe static strain will select some particular point where some detect in material or workmanship exists, and this imperfection when found is assigned as the cause of the failure, even though the defective part may have been amply able to withstand normal potential.

Still another fact renders the tracing of static strains difficult. Only occasionally are the conditions favorable for the exciting spark to be followed by the normal e.m.f. of the circuit, which alone makes the spark detectable. That means that if a watch is kept for the effect of any particular cause of static, such as switching, perhaps nine times out of ten this effect will not be discovered, because it is not made evident by the generator e.m.f.

Sometimes a short circuit may be started by a static spark which involves so few turns of the coil or has so much resistance that the fuses and instruments will not be noticeably affected, and nothing is known of the trouble until it has developed, which may require some minutes or some hours.

I do not mean to give the impression here that I consider every failure of high tension apparatus to be, somehow or other, due to static causes, for such is far from the truth; nor that such difficulties are of everyday occurrence, for this would be much further from the truth; but it is true that static strains do cause breakdowns, and that such are very difficult to trace to their true causes.

\section{Protection from Static.}

Protection from static is a question of growing importance, for several reasons. Circuits of high potential and long distances are rapidly extending, both as regards number, size and potential. The troubles due to static are certain to be more commonly recognized. In the future the standard of service will gradually rise and nothing threatening its interruption will be tolerated.

I propose, in what follows, to describe a system of protection of high tension apparatus from static strains that is applicable to practically all cases of high tension transmission. In actual plants difficulties from static are found to occur usually in generators, transformers or instruments, connected directly to the 
high tension circuits, or between lines, or to ground at ends of lines or other reflecting points. Direct strokes of lightning are an exception to the statement regarding the location of damage. Why strains should appear at these points has been already explained.

These injuries are of two distinct classes, short circuits and grounds, each type of injury being caused in a different way and requiring its own protective apparatus. They will be treated separately.

\section{Protection against Short Circuits.}

As already fully explained, static causes short circuits by producing a momentary concentration of potential (which may or may not be greater than normal line potential) upon a few layers of the coils, which very probably may have been intended to withstand only a small part of the strain brought upon them. This excessive momentary strain results from a very abrupt change of potential of the terminal or other point of the coil, either up or down. With such a change, until charge can flow to the more distant layers, the full voltage of the potential change will be impressed on the layers of the coil between the point of application of strain and those points to which charge has not penetrated. If this voltage is too much for the strength of the insulation, a spark passes. If conditions are favorable, the e.m.f. of the generator causes a current to follow, and a burnout will usually result. In some cases, however, current from the generator may start to follow and drop out at the end of the first alternation.

The characteristic of the static wave which causes the static strain to be concentrated on a few layers of the coil is its abruptness. Therefore, if this wave be forced to pass through a choke coil and its abruptness thus reduced, the strain on the coil will be lessened; if the choke coil be sufficiently powerful, the wave will be rendered harmless. It must be noted that it is by no means necessary for the choke coil to be effective, that it be made powerful enough to remove entirely the strain from the transformer coil; for the latter's insulation will withstand a much higher e.m.f. of a static nature which exists but an instant than of a continuous nature like that impressed by the generator. Also, there is usually allowed (perhaps on account of the results of previous experience with static) a very great margin in the insulation between the layers of coils.

The exact amount of abruptness allowable in the wave will 
depend on circumstances. Looking at the question more closely, it is seen that a choke coil cannot be said to have a definite protective power regardless of the apparatus it protects; for more choking effect is required to protect a transformer coil of many turns of layers than a coil of few. The condition to be fulfilled is that by the time maximum voltage is reached at the transformer terminal, charging current shall have penetrated far enough into the transformer coil so that a sufficient number of layers shall share the strain for their combined insulation strength to be able to resist it. The function of the choke coil is thus seen to be simply one of delaying the wave to give the inflowing charge time to become distributed over the coil. The length of time required depends on the rate at which the charge penetrates the coil and the strength of the insulation. The inductance of the protecting choke coil should be larger than that of the minimum portion of the transformer coil which is able to stand the strain of the most severe static wave that may occur.

But such a choke coil has other effects than the retarding of static waves; it will also retard the normal flow of current; in some cases to such an extent as to be quice an undesirable feature. The choke coil also has resistance and will waste energy, and unless a prohibitive cost of copper is used, this loss of energy will sensibly affect the efficiency of the plant; further, the coil will get too hot. It will be found in most cases that with high tension apparatus choke coils powerful enough to protect perfectly are impracticable on account of size or cost.

\section{Static Interrupter.}

To meet this very serious difficulty the author proposes the use of a device he has called the "static interrupter." The "static interrupter" is a combination of a choke coil and a condenser connected in such a way as to magnify the effect of the choke coil as regards static waves without causing a sensible derangement of the normal operation of the circuit. By the use of this device a comparatively small coil can be made to afford the same protection as a coil of much larger dimensions without entailing either excessive cost or excessive loss of energy or heating.

The static interrupter consists of a choke coil in series with the line and a condenser connected by one terminal between the choke coil and the winding to be protected and by the other terminal to such parts of the apparatus as have e.s. capacity with this high tension winding; in a transformer to the low tension. 
winding and the core. However, as these parts are always (as compared with the high tension) at ground potential, the second condenser terminal may be connected directly to ground. The diagram of connections for the interrupter protecting a transformer or generator is shown in Fig. 7 .

\section{Protective Power of Static Interrupter.}

It is evident that more time is required for sufficient charge to pass the choke coil to charge the transformer terminal and condenser together than would be required to charge the terminal alone. This means that the maximum static strain will be distributed over a larger number of layers in the first case than in the second. The ratio of the e.s. capacity of the condenser to that of the transformer terminal and the adjacent parts of winding is an important factor in determining the protective power of
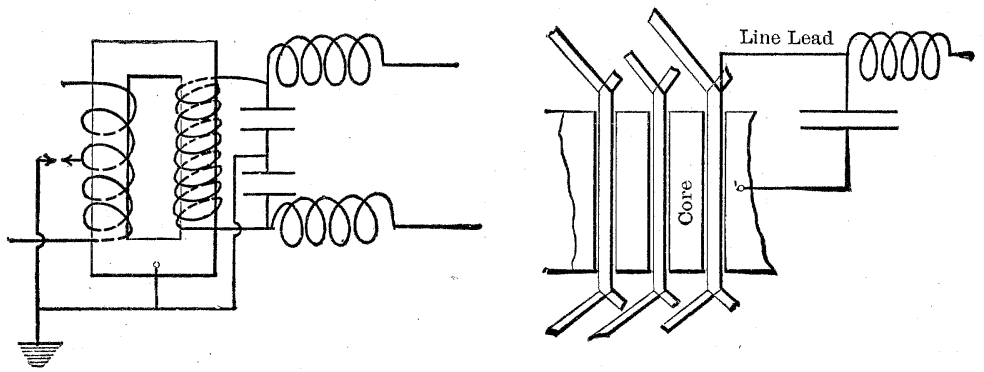

FIG. 7.-Static Interrupter Protecting a Transformer and High Tension Generator.

the interrupter, as it indicates the extent to which the potential of the terminal at any instant is reduced by the addition of the condenser. It is possible to get almost any desired degree of protection by the use of a sufficiently large condenser without unduly increasing the size of the choke coil in series with the circuit. Actual experiment shows this method of protection to be very effective in reducing the static strains in coils.

On the other hand, the use of the static interrupter, although it delays the penetration of the crest of the advancing static wave, does not necessarily prevent the ultimate rise of potential of the terminal, that is, it affords no direct $F$ rotection against grounds. However, in cases where the interrupter is used in connection with a lightning arrester (which should always be connected on the side of the choke coil farthest from the condenser) the delay of the wave does give additional protection against grounding of 
the winding, as it allows the arrester more time to discharge the line.

The interrupter performs a more important function in connection with the discharge of an arrester by protecting the winding from the violent strains that result from the abrupt grounding of the circuit caused by the discharge. As the discharge of the arrester occurs only when line potential is much above normal, and may be carried momentarily much beyond ground potential on account of its oscillatory nature, the shock to the coil may be a very severe one.

\section{Location in Circuit of Static Interrupters.}

It is thus evident that by placing a proper static interrupter in each lead wire of a transformer or other apparatus which may be connected with a source of static disturbance such as a line or a high tension switch, complete protection may be secured against short circuits in the windings caused by static originating outside of the transformer. As switching is a frequent cause of static strains, the interrupters should in general be placed between the apparatus and the switches thus making the interrupter virtually a part of the transformer or generator to be protected and not a part of the line. If, however, each individual transformer is provided with static interrupters a larger number of interrupters is required than would be necessary for protecting a bank of transformers as a whole from disturbances upon the transmission line. If there is no switching of individual transformers it is then satisfactory to arrange the apparatus so that several transformers will be protected by the same interrupters. For example, in polyphase systems, a group may be protected as a unit instead of giving individual protection to each transformer. The precise arrangement of apparatus in a given case will depend upon the number of transformers to be protected and the arrangements which are provided for switching.

There are certain cases in which switching may be done between the interrupter and the transformer. The transformer may be cut out of circuit by the opening of circuit breakers, fuses or switches, or transformers may be connected to a live high tension circuit, provided their low tension windings are at the time supplied with current from the same generator as the circuits from which they are switched. Such switching causes no change of potential at the terminals and consequently no strain.

Also fuses and circuit breakers may be opened by overloads on 
short circuits without regard to the low tension connections or without danger from static as the arc formed assures a gradual change of potential.

The proper connections for static interrupters in the arrangements of apparatus most frequently found are shown diagrammatically in Fig. 8 .

\section{Features of Commercial Static Interrupters.}

Some of the features of actual static interrupters intended for commercial service work may be of interest. These interrupters

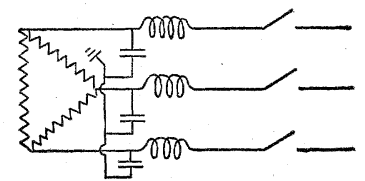

Three Static Interrupters Protecting Three Transformers Connected in Delta.

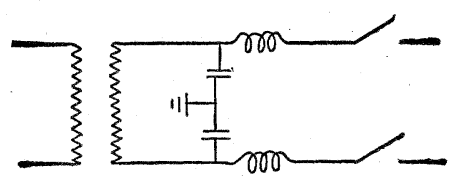

Two Static Interrupters Protecting One Transformer.

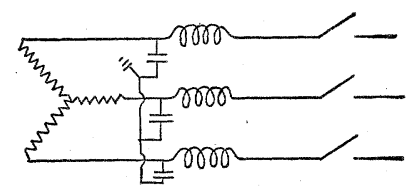

Three Static Interrupters Protecting Three Transformers Connected in Star.

Three-phase Generators'

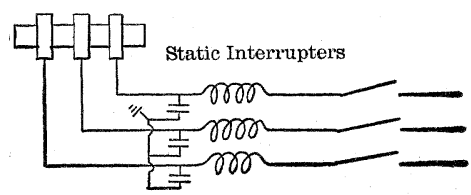

Three Single Pole Static Interrupters Protecting a Three-phase Generator.

Single-Phase and Two-Phase Generators are protected by Two and Four Single Pole Static Interrupters Respectively, Connected in a Similar Manner.

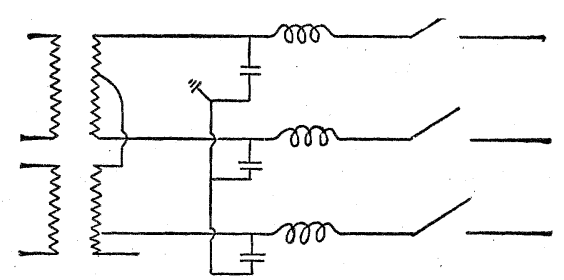

Three Static Interrupters Protecting Two Transformers Connected for Three-Phase High Tension and Two-Phase Low Tension Circuit.

FIG. 8.-Diagram Showing Arrangements of Static Interrupters Protecting Generators and Groups of Transformers.

are usually single pole; that is, one coil and one condenser are placed in a containing case. As the interrupters are used on high voltages, coils and condensers are placed in oil. The oil facilitates the cooling as well as strengthening the insulation. The choke coils, which contain no iron, receive the full force of the static waves which they are intended to check, and are very heavily 
insulated. On a three-phase circuit the condensers must support continuously a strain approximately 6,10 of the normal line voltage without breakdown and without overheating; they must also be able for moderate lengths of time to stand full line voltage in case one leg of the circuit becomes grounded.

The fact may not be generally appreciated that condensers have a very considerable heat loss which must be carefully considered in designing the interrupter. This loss for any given dielectric varies in actual condensers approximately as the square of the voltage, and increases rapidly with the temperature. The loss in a condenser may be increased several times by changing its temperature from $20^{\circ} \mathrm{C}$. to $100^{\circ} \mathrm{C}$. This fact indicates the importance of not allowing condensers to get hot.

The actual e.s. capacity of condensers for static interrupters is made much larger than the e.s. capacity of the windings they are to protect, but is still very small-seldom exceeding a few hundredths of a microfarad. The reaction of the condenser upon the circuit for generator frequencies is therefore negligible.

At the end of this paper are shown some views of a static interrupter for 50,000 volts, 19 amperes.

\section{Protection Against Grounds.}

A ground is the result of a rise of potential higher than the insulation of the apparatus is adapted to withstand (assuming that the insulating material contains no local defects). Such an excessive strain may result from static disturbances or from other causes. It has been shown that lightning may be one cause, and that under certain circumstances switching and similar disturbances may produce high momentary voltages. Therefore, unless the apparatus is insulated to stand such excessive potentials, which may be possible with low and moderately high voltage circuits, but is hardly practical with extra high tensions, we must use some means of prevention. The standard lightning arrester spark gap used to prevent excessive potentials from lightning is effective also against the other static causes.

\section{Lightning Arresters.}

A simple air gap between line and ground is an ideal lightning arrester or line discharger as far as its protecting power is concerned. It absolutely resists the passage of current up to a certain voltage, which can be predetermined quite closely, and if this critical voltage is exceeded, breaks down and furnishes a noninductive and nearly resistanceless discharge path for carrying 
off any amount of static electricity that may appear on the line. This means that when once the gap has broken down no amount of charge on the line can raise its potential above the ground. However, if the ground connection be not perfect or if either in the arrester or in its connections there is sufficient inductance or resistance, it is then possible to have a dangerous rise of potential on the line after the air gap has broken down due, of course, to the checking of the discharge of the line by the inductance or resistance.

Unfortunately, however, when single spark gaps are used as lightning arresters, their simultaneous discharge on two or more legs of a line forms a short circuit, and the usual result follows. If one leg of a circuit is accidentally or permanently grounded an arrester discharge on any one of the other legs causes a short circuit.

The air-gap lightning arrester to be commercially successful, in addition to giving protection from static must be able to interrupt the short circuit current which tends to follow lightning discharges. The devising of an arrester to meet all conditions is a very difficult problem. Fortunately, however, a large number of small gaps in series may be substituted for one single gap of larger dimension which greatly increases the "non-arcing" power. A very few gaps of non-arcing metal are satisfactory under some conditions; but the more the gaps are sub-divided the more effective the arrester. On high voltages, however, and under some other circumstances, such a large number of these gaps must be used on the arrester, as to interfere with its protective power by requiring a rather excessive voltage to cause the gaps to discharge. If the amount of the current that can flow on short circuit be limited either by series resistance or otherwise, a considerably less number of gaps will be non-arcing.

\section{Series Resistance.}

The use of series resistance to render an arrester non-arcing has the advantage of cheapness and simplicity, and is very effective as far as rendering the arrester non-arcing is concerned, but it has the great disadvantage of introducing the resistance in the path of the discharge of the line. In many plants the small size of the generators limits the current flow on short circuit in such a way as to render no series resistance necessary. It must be remembered in this connection, that all synchronous apparatus connected to an electric circuit (and sometimes induction motors), will temporarily act as generators at the time of a lightning dis. 
charge, on account of the energy stored in the momentum of their revolving parts.

In many cases where the short circuit current taken by the arrester is not excessively large, the series resistance may be whunted by additional gaps which will allow the static discharge to pass directly to earth around the resistance. If the parts are properly proportioned, the subsequent arc will be arawn from the shunted gaps by the resistance and thus extinguished. If the number of shunted gaps is no greater than of the unshunted gaps, no greater voltage will be required to discharge over the whole number than over the unshunted gaps alone. This is because the shunted and unshunted gaps are separately broken downone after the other. Therefore, no protecting power is lost by the addition of the shunted resistance.

In the cases where such resistance, shunted by gaps, is not sufficient, a small series resistance in addition will make the arrester non-arcing. In other words, where a large series resistance is required, part of it may be shunted by gaps in such a way as to introduce only a small resistance in the discharge circuit of the arrester.

It should be noted, as in the case of short circuits in coils from static sparks, that if the discharge through an arrester passes at or near the time of zero e.m.f. of the generator, there will be little likelihood of a permanent short circuit.

Although the insertion of resistance in the discharge path of an arrester may under some circumstances prevent the very thing that the arrester is supposed to accomplish, nevertheless its great effectiveness in rendering the arrester non-arcing and its wide commercial use requires a statement of the conditions under which it may safely be used. It evidently makes no difference how much resistance or inductance is used in series with an arrester, provided it is always able to discharge the electricity as fast as it actually appears on the line at the point where the arrester is connected, without causing a potential in the arrester greater than that required to cause the arrester to discharge in the first place. In discharging electricity, a resistance requires a potential equal to the product of the current strength and the resistance; inductance causes a counter e.m.f. equal to the product of its inductance and the rate of increase of the current. At each instant of time the current flowing in the arrester circuit has a definite value and a definite rate of increase (or decrease). Therefore, for the arrester to meet the above condition, the sum 
of the two potentials caused first by the current at the moment flowing in the resistance and second by the rate of increase of current in the inductance, must at no time exceed the potential required to cause the arrester to discharge.

It will be readily seen that the greater the quantity of static electricity to be discharged and the more sudden its appearance, the more severe the conditions on the arrester. Also that the greater the resistance and the inductance of the arrester circuit the greater the likelihood of a dangerous rise of potential, Just what the greatest allowable values of resistance and inductance are in any given case will depend upon various circumstances and cannot usually be definitely predicted. Therefore, in all cases the lowest practicable amount of resistance and inductance should be used in series with the discharge path of the arrester. It should be noted that with an increasing current flow through the arrester circuit the quantity of electricity discharged by the arrester before the dangerous point of potential is reached will be much more than proportionally greater with a low than with a high resistance. That is, if the series resistance in one case is one-half that in another, both discharging the same increasing current, the quantity of electricity that will have passed the first arrester when a given potential is reached is much more than twice that passed in the second case, when the same potential is reached. This fact makes it still more desirable that series resistances should be kept as low as possible.

Resistance and inductance in series with the arrester circuit have the advantage of preventing to some extent the very sudden grounding of the line that otherwise results from the arrester discharge and which causes severe strains in the windings of adjacent apparatus. This is an advantage by no means to be overlooked, for it will be remembered that short circuits are much more frequent than grounds.

\section{Location of Arresters.}

A proper air gap lightning arrester will prevent an excessive rise of potential above the ground at that point of the line to which it is connected, but on account of the wave nature of the static disturbances it will not necessarily prevent an excessive rise of potential at some other point of the circuit more or less distant. For if the arrester happen to be at the zero point of a wave, the arrester will not discharge, even though the crest of the wave at some other point may be at a very high potential above the earth. 
It is a somewhat difficult problem to obtain complete protection at all points; however, in actual circuits adequate protection can usually be secured. Actual commercial plants fall into two classes, viz.: plants in which the apparatus connected to the high tension circuits is scattered about at numerous more or less widely separated points, and plants where the apparatus is all concentrated at a few points:

\section{(a) Plants with Apparatus Distributed.}

For absolutely complete protection from ground in plants having distributed apparatus, arresters will have to be placed wherever there is apparatus that needs protection. In most cases this would mean an almost prohibitive expense. Fortunately such plants are ordinarily of comparatively low voltage, and therefore require protection (as far as static from grounds is concerned) only from lightning. Therefore, by placing arresters in such locations as readily to discharge static waves that may arise in the most exposed portions and by scattering arresters at intervals over the line at other points sufficient protection is secured. The exact number of arresters required in any given case depends upon the local conditions. This type of circuit, with distributed apparatus, is seldom found above 3,000 volts. (b) Plants with Apparatus Concentrated at a Few Points.

In these cases the sources of static disturbance are either at some point away from the apparatus or are, electrically speaking, at the point where the line enters the station; that is, when a rise of potential is caused by switching in the station, all the high tension wiring is at the same potential and the rise can be limited by an arrester placed on each leg of the line at the point where the line enters. But these arresters, provided they be placed on the line side of apparatus, will also protect from dangerous static waves produced at points away on the line, for such waves must pass the arrester before they can reach the apparatus, and will thus cause the arrester to discharge. Therefore, in circuits of this type, which includes practically all high tension circuits, protection from grounds will be secured by proper arresters, placed one on each line wire where it leaves a station. The arrester must be placed outside of all choke coils and static interrupters, so that these may prevent static strain in the apparatus from the discharge of the arrester. This distribution of arresters is the one that has usually been followed in actual installations. 
The system of protection outlined for high tension circuits, therefore, consists of the use of proper lightning arresters at appropriate points to protect against grounds, and static interrupters to protect apparatus against short circuits. The lightning arresters should be of adequate design, but no radical change from the usual practice in regard to location is required.

The use of the static interrupter is, however, new, and it has been one of the objects of this paper to give a full exposition of the protection it affords and to discuss carefully the conditions which render its use advantageous.

\section{SuMMARY.}

A summary of the most important conclusions regarding arresters and interrupters follows:

(1) Damage resulting from static strains is of two sorts: short circuits and grounds. It is the function of the static interrupter to protect apparatus against short circuit strains; it is the function of the lightning arrester to relieve the line of excessive static charge and thus prevent grounds.

(2) The static interrupter consists of a choke coil and condenser, the latter being connected between line and ground on the apparatus side of the choke coil. The interrupter delays and smoothes out static waves which attempt to pass it. The condenser, by absorbing a large part of the static charge which passes the choke coil, very much increases the effectiveness of the coil.

(3) The choke coil and condenser of the interrupter must be adapted to the circuit upon which it is to be run, and must be proportioned with regard to the design of the apparatus it is to protect. The interrupter should be placed nearer the apparatus. than any switches which are to be operated with potential on the line. (See page 147 for exceptions.) The interrupter is thus essentially a part of the apparatus to be protected and not a part of the line or the arrester.

(4) Lightning arresters must be able to discharge electricity from the line as fast as it actually appears there, and must also be non-arcing under all conditions. Any resistance or inductance used in series with the arrester should be low enough to allow the passage of all actual discharges without causing excessive potential on the arrester.

(5) On the systems where the apparatus is distributed the arresters should also be distributed. 


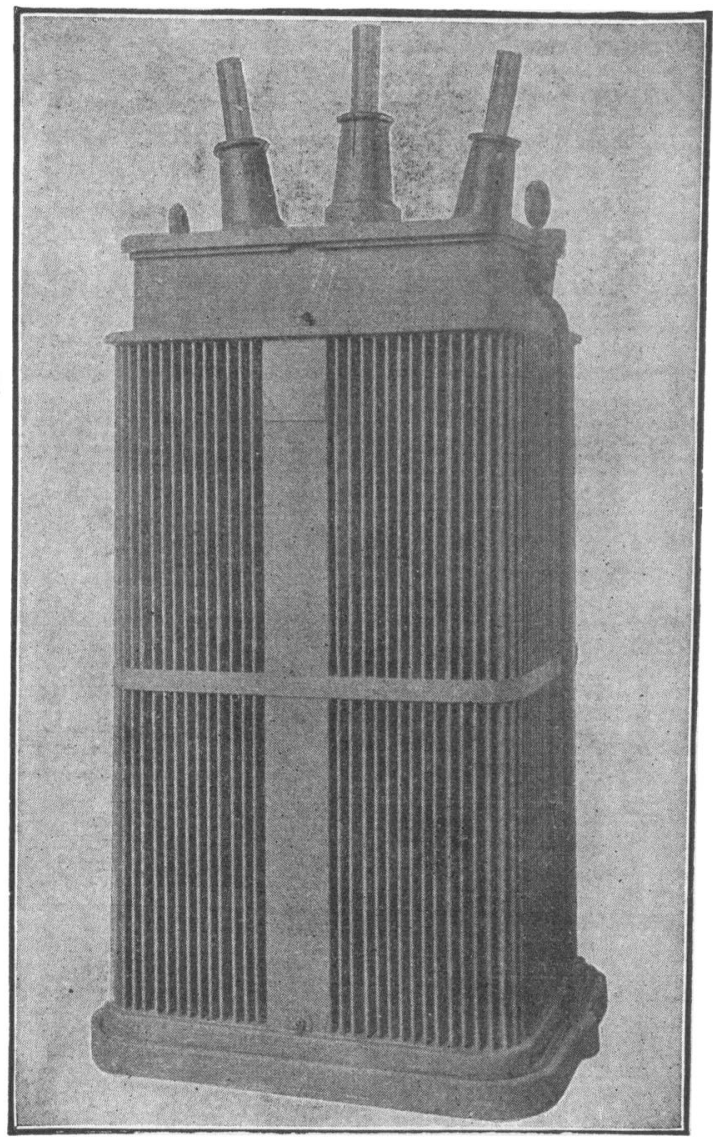

Static Interrupter with Case Complete. 


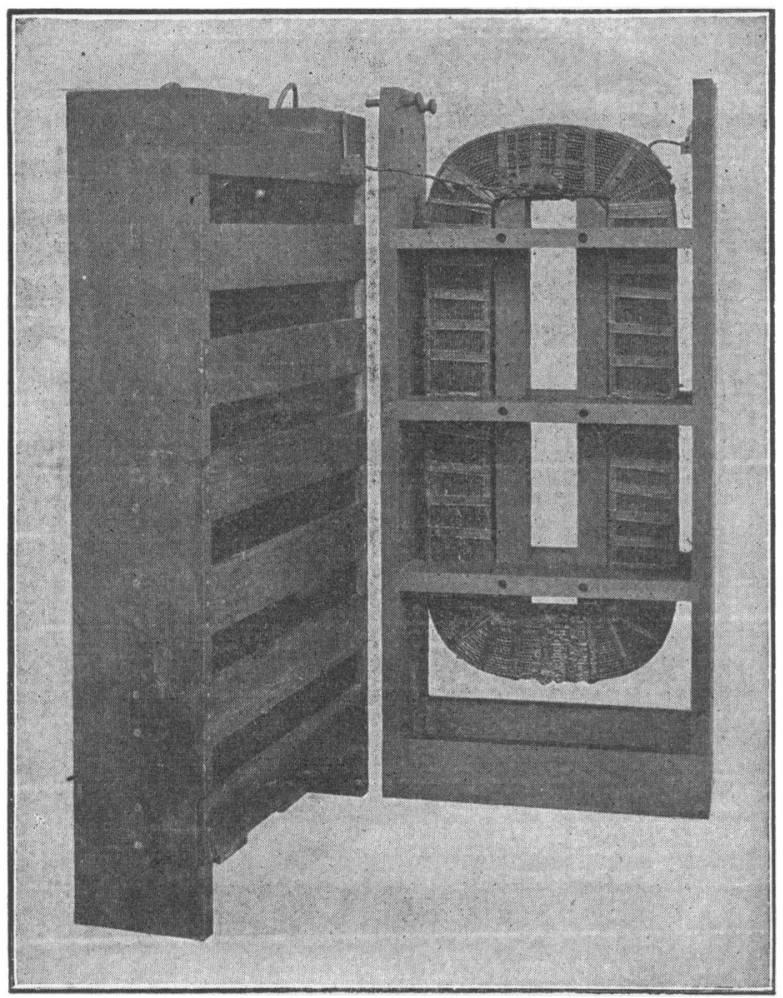

A Static Interrupter Open.

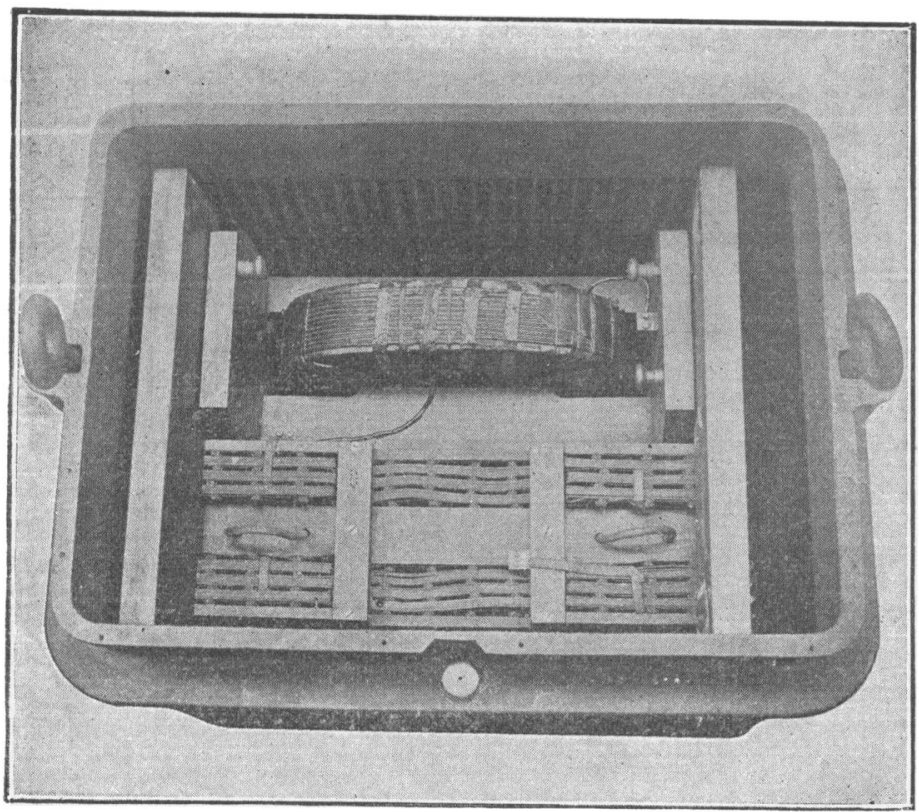

View of Static Interrupter from the Top. 
On systems with apparatus concentrated at a few points arresters should be placed on each line wire where it leaves a station.

(6) Well designed static interrupters and lightning arresters, properly arranged, furnish a complete system of protection against the static disturbances found on high tension lines.

It is quite, evident that the protection of the apparatus of a large high tension system, with its numerous opportunities for static disturbances and its widely spread apparatus, is by no means a simple problem, to be solved off-hand by the use of a few lightning arresters of any available style. The type and location of the protective devices must be adapted to the actual conditions of the plant; the severity of the lightning in the neighborhood, the voltage of the system; the type of apparatus to be protected and perhaps the actual constants of the circuits. The determination of the most satisfactory protection is a matter to be arrived at from a combination of theory, experience and experiment, and any protective system should be freely modified when actual trial shows a better arrangement. It is to be hoped that engineers and others who have the opportunity will carefully stidy experimentally the questions of static, giving them all the attention that their importance deserves, and that we may in consequence look forward to the time when difficulties from static causes may be practically unknown.

\section{Appendix I.}

EXPERIMENTS ILLUSTRATING LOCAL CONCENTRATION OF POTENTIAL IN A HIGN-TENSION COIL.

Shown at the meeting of the Institute, February 14th, 1902.

To illustrate the effect of switching short-circuits and lightning in producing local concentration of potential in coils connected to a transmission line, experiments were performed as follows :

1. Effect of Switching-Charging a Dead Transformer from a Live Line.-The arrangement of the apparatus is shown in Fig. 9.

A $30 \mathrm{k}$.w. 30,000 volt raising-transformer was used to charge the line. The electrostatic capacity of the line is represented by a pair of oil condensers (c.c.) connected in series with their common terminal grounded. Each of these condensers had a capacity of approximately .012 microfarads. The ratio of transformation of the raising-transformer was such as to give approximately 25,000 volts on the line.

The transformer coil was a single coil of 52 layers surrounded 
by a heavily insulated metal plate $\mathrm{D}$, which represented the low tension winding and the core. This coil was taken to be the coil of a lowering-transformer lying next the terminal at which switching was to be done. The remainder of the transformer, which is permanently connected to the other line, is replaced by the high resistance $\mathrm{R}$, which serves to support the line voltage when the circuit is closed.

The blunt-nose spark-gap $\mathrm{P}$ was used to measure the maximum instantaneous voltage on different parts of the coil caused by switching. Leads were brought out at various points in the coil to facilitate this measurement.

In the experiment the switch $\mathrm{A}$ was closed and opened repeatedly until the measuring spark-gap could be so adjusted as to indicate the maximum instantaneous voltage caused by closing the switch; this point was indicated when the gap would break down

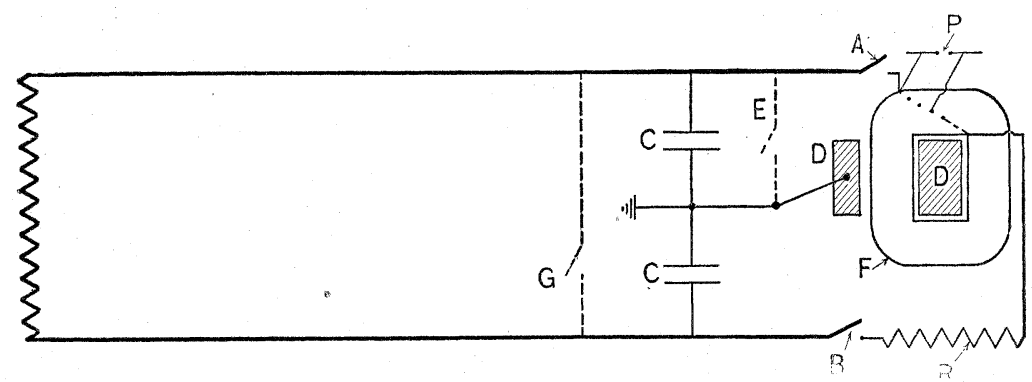

Fig. 9.-Diagram of connections to show effect of charging dead transformer and effect of short circuits.

once or twice only in 50 trials. A large number of trials is always necessary, as the closing of the swing-switch does not always occur at the point of e.m.f. wave most favorable for causing a high potential.

By this means the maximum potential between the terminal and the 4th, 8th, 12 th layers, etc., was successively determined. The results are plotted in a curve in Fig. 10. The normal voltage that would exist on a layer, were the coil in an actual transformer, is something like 75 volts. The maximum potential reached between the lines during switching was approximately 33,000 volts. This is a very considerable increase over the 25,000 volts of the line, and represents the rise of potential usually accompanying the charging of a condenser through inductance. It is evident that very great strains are produced by the switching on the layers next the terminal. 
2. Effect of Short Circuits.-The same apparatus was used, and with the switch A closed, the line was repeatedly short-circuited by a switch connected at G. Measurement of potential on the various layers of the winding, made in exactly the same manner as before, gave voltages approximately one-half of those due to switching, shown in Fig. 10. As the change of potential of the terminal in the case of short-circuiting is only one-half of that caused by charging the dead transformer, only half the instantaneous rise of potential is to be expected.

3. Effect of Lightning.-The apparatus of the test which is very similar to that used in the above experiments is shown in Fig. 11. The condenser represents the electrostatic capacity between a charged cloud and the line, and the closing of the switch $H$ the discharge of the cloud. This arrangement is not strictly analogous to the effect of lightning on a line, as dynamic induction

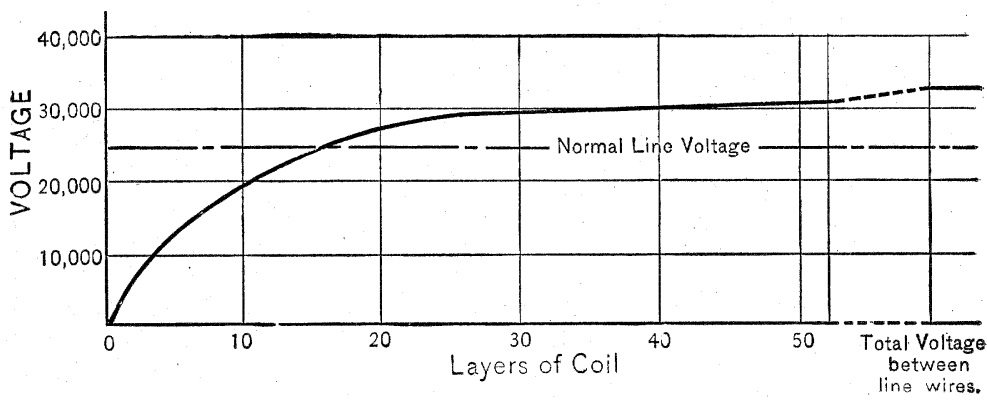

FIG.10.-Curve showing concentration of potential on layers of coil.

is not represented, but the effect of dynamic induction would be exactly similar to that of static induction, which is shown, so no error is introduced. Tests of the instantaneous voltages in the coil, as before, showed a curve practically identical with Fig. 10.

These tests show what the analysis in the paper requires, that abrupt static changes cause a local concentration of potential near the terminals of the coils, the amount of which depends simply upon the abruptness and severity of the potential change.

To illustrate the protective power of choke coils and statio interrupters experiments were performed as follows:

1. Choke Coils. - Three choke coils were used, all having a mean length of turn about two feet. The first had 80 turns, the second 352 turns, the third 1,200 turns. With the coil of 80 turns placed in the lead of transformer coil next to switch $\mathrm{A}$, the instantaneous voltage on the first four layers, which was caused 
by switching with no coil in the lead, was cut down about onethird. But this coil had no appreciable effect on the instantaneous voltage on the first 30 layers, showing that a few lavers of the transformer coil may be much more easily protected than many layers.

With the coil of 352 turns connected in the lead, however, the voltage on the first 30 layers was cut down to nearly one-quarter its original value. With the coil of 1,200 turns the abnormal voltage was cut down to less than one-tenth of its original value. Thus the effectiveness of a choke coil in reducing the shortcircuit strains is clearly demonstrated.

2. Static Interrupters. - It had previously been shown, as described above, that the choke coil of 80 turns could not appre-

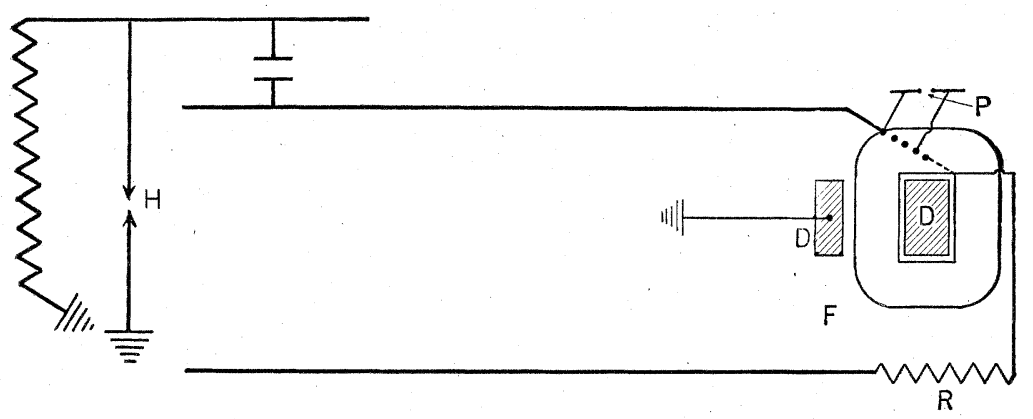

FIG. 11. - Diagram of connections to show effect of lightning.

ciably lower the instantaneous voltage on the first 30 layers of the transformer coil. When, however, a condenser of less than .0015 microfarads capacity was added, so as to form a static interrupter, this abnormal e.m.f. was reduced nearly 50 per cent. and with a larger condenser was cut down to approximately onequarter of its original value. A still larger condenser would have practically removed the abnormal potential altogether. Or if the voltage in the first 15 layers had been measured, instead of in the first 30 , it would have been found a few per cent. of its priginal value.

It is possible by properly proportioning coil and condenser to get any desired reduction in the abnormal voltage on any desired number of layers. 
Appendix II.

MECHANICAL MODEL ILLUSTRATING THE PROPAGATION OF STATIC WAVES IN A LONG TRANSMISSION LINE.

Exhibited at the meeting of the Institute, February 14, 1902.

To illustrate the propagation of static waves in a long transmission line, such as result from abrupt changes of static potential, the mechanical model described below was exhibited. A long transmission line (or cable) may for all practical purposes be represented by a series of choke coils and condensers as in Fig. 1, page 119. The law governing the currents, charges and e.m.f.'s in this circuit may be stated as follows: At any instant the difference in potential between any two neighboring points is equal to the e.m.f. consumed by the passage of the current through the resistance of that portion of the line wire between the points, added to that consumed in the back e.m.f. due to the inductance of the line between the points. If any mechanical device can be found, such that the same law will govern the motion of its parts, then exactly the same sort of waves will be produced in one case as in the other, provided appropriate forces are applied. The model exhibited, which is shown in the cut, Fig. 12, is such a device.

Referring to Fig. 1, page 119, the condensers representing the capacity to ground at the points B, C, D, etc., are replaced in the model by the springs seen near the top of the frame. The condensers representing the capacity between line wires are replaced by the springs shown near the bottom of the model. These springs are not so large as the first, as so much extension is not required. There are two sets of springs of both sizes, indicating the two line wires; one set is hung on the front of the frame, the other on the rear. The inductance between two adajcent points of the line is represented by the inertia of the lower line of pulleys, which are controlled by the small springs. These wheels are made thick to give them mass. The springs representing capacity and the heavy wheels representing inductance are connected together by an endless thread which serves the function of a conductor between points. As this thread must move freely it is carried on pulleys; it must not slip, however, on the heavy pulleys. The resistance of the line is represented by the iriction of the wheeis and the stiffness of the thread. It must be noted here that the model is not strictly analogous to the electric circuit in tris respect, for its friction is not strictly 
proportional to the velocity of motion, as it should be, since the loss of e.m.f. due to resistance is exactly proportional to the current. However, as the friction is relatively small, the movements of the model are almost wholly controlled by the springs and the inertia; the resistance simply causes a dying out of the waves.

The two divisions (front and back) of the model representing the two line wires are connected together through the heavy wheels at the bottom, which are rigidly linked in pairs by cords running over stationary pulleys. A motion upward of one heavy wheel means an equal motion downward of its mate. That is, equal positive and negative charges must appear simultaneously in the two transmission wires. As the lower springs represent the capacity between the line wires, their extension measures that part of the total line charge due to this capacity, and at the same time indicates the voltage between lines. Similarly, an extension of the upper springs indicates that portion of the total line charge due to the capacity to ground, and, as well, the potential rise above the earth. Motion or displacement of the thread which runs over all the pulleys indicates quantity of electricity. Velocity of motion represents current strength. Force or tension on the cord represents e.m.f. and the device used for producing this force, the generator. The cord runs from one line to the other through the generator, so that whatever current flows out at one terminal must flow in at the other. To represent the lines short-circuited, at the farther end the cord passes freely from one line to the other. To represent an open circuit in the line at any point, it is necessary only to hold the thread rigidly at the point where the open circuit is supposed to exist, so that no electricity can pass. Then, releasing the thread is the equivalent of closing the open circuit.

The generator consists of a pulley carrying the endless thread supported by two loops of cord running in proper grooved wheels, as shown at the right in Fig. 12. These loops or belts hang on a revolving rod driven at a high rate of speed by a motor, so that they slip continuously on the rod. The pulley and grooved wheels, which are fastened rigidly to the same shaft, are free to turn by the slipping of the belt. The friction of the belts on the revolving rod causes a constant torque on the pulley carrying the thread, and consequently on the adjacent parts of the line. This torque acts as an e.m.f. As the rod supporting the belts revolves at a very high rate of speed, the comparatively 
slow motion of the driving pulley hanging below will not materially affect the friction. Therefore, the e.m.f. is constant regardless of the " current" flowing (that is, regardless of the velocity of the thread. The amount of the e.m.f. applied is varied by hanging weights on the driving pulley to increase the friction of the slipping belts on the supporting rod. These weights must, of course, be supported through bearings in which the driving pulley may revolve.

In the model, the energy stored in capacity is represented by

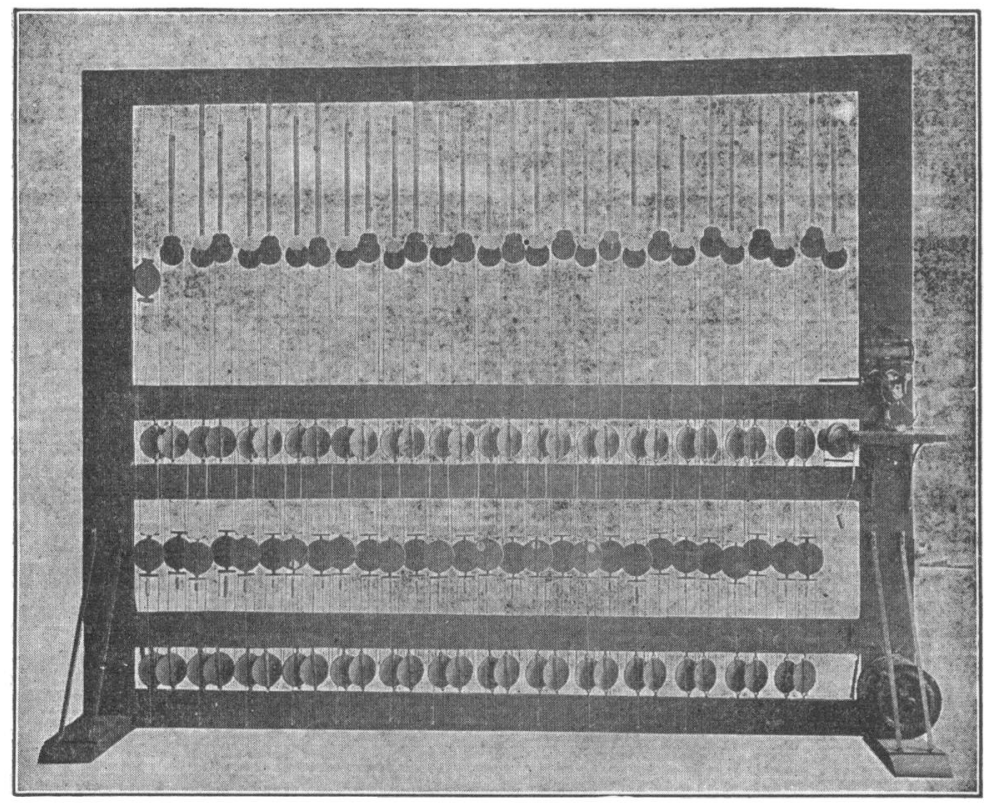

Fig. 12.-Mechanical Model of Transmission Line.

the energy stored in the stretched springs; and the energy stored in the inductance is represented by the kinetic energy of the revolving pulleys. When a steady direct-current flows through the line storing magnetic energy in its inductance, all the wheels have a constant velocity and a constant kinetic energy

The following well-known phenomena in a transmission line were illustrated by the model: Charging a dead open-circuited line; short-circuiting a charged line at the farther end; opening 
a short-circuit at either end; ohmic drop in the line; energy stored by direct current in the short-circuited line.

Several seconds are required for a wave to pass the length of the model and return, The length of time required depends on the moment of inertia of the wheels and the elasticity of the wheels. Each heavy wheel weighs six ounces and is approximately $5 \frac{1}{2}$ " in diameter. Each of the large springs has such an elasticity as to extend $26^{\prime \prime}$ under the weight of one-half pound. The small springs extend $10^{\prime \prime}$ under the same strain. It is important that the friction be made as small as possible. 